University of Redlands

\title{
A New World Map on an Irregular Heptahedron
}

A Major Individual Project submitted in partial satisfaction of the requirements for the degree of Master of Science in Geographic Information Systems

By

Nathan Dennis McCall

\author{
Mark Kumler, Ph.D., Chair \\ daan Strebe \\ David A. Burrows, M.A.
}

December 2006 
A New World Map on an Irregular Heptahedron

Copyright (C) 2006

by

Nathan Dennis McCall 
The report of Nathan Dennis McCall is approved.

Qenvolystrite daan Strebe

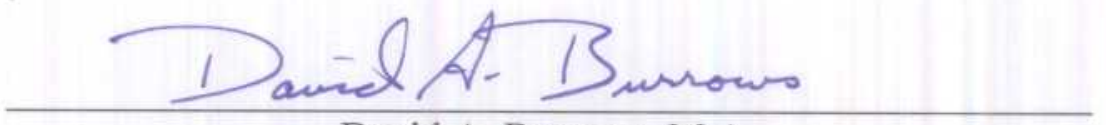

David A. Burrows, M.A.

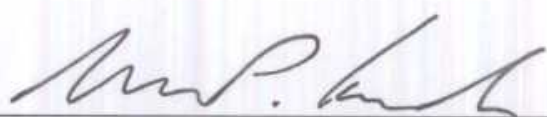

Mark Kumler, Ph.D., Chair

December 2006 



\section{ACKNOWLEDGEMENTS}

I would like to thank daan Strebe, David Burrows, Melita Kennedy, Dr. Waldo Tobler, Dr. Mike Bloxham, and Dr. Sandy Koonce. I would also like to acknowledge Dr. Mark Kumler for his unyielding confidence when many others, including myself at times, thought I was crazy for attempting this project. 



\begin{abstract}
A New World Map on an Irregular Heptahedron

By

Nathan Dennis McCall
\end{abstract}

Using polyhedral approximations of the globe for the purpose of creating map projections is not a new concept. The implementation of regular and semi-regular polyhedra has been a popular method for reducing distortion. However, regular and semi-regular polyhedra provide limited control over the placement of the projective centers. This paper presents a method for using irregular polyhedra to gain more control over the placement of the projective centers while maintaining the reduced distortion quality found in polyhedral projections. The method presented here uses irregular polyhedra based on gnomonically projected Voronoi partitions of the sphere. 



\section{Table of Contents}

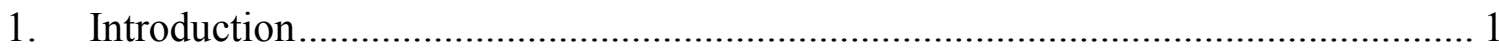

1.1. A perspective projection of the globe onto a plane........................................... 1

1.2. Uninterrupted projections used for world maps................................................ 3

1.3. Interrupted projections used for world maps ................................................... 8

1.3.1. Goode's interrupted homolosine ........................................................ 8

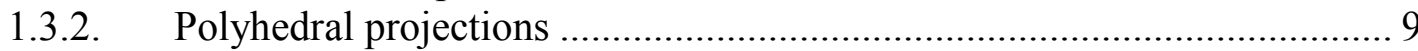

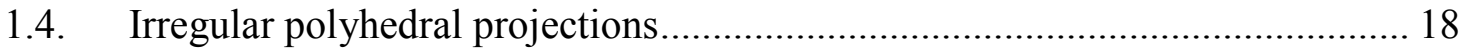

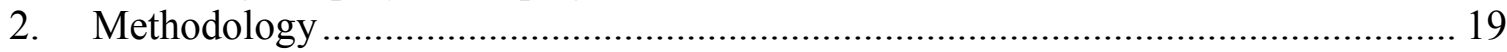

2.1. The generating points (projective centers)................................................... 19

2.2. The polyhedron edges ............................................................................. 19

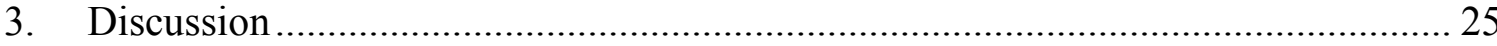

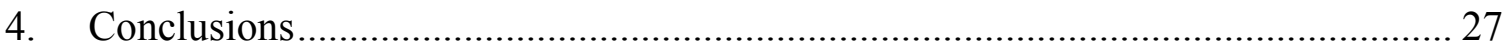

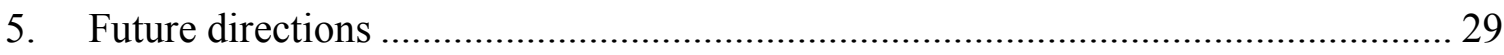

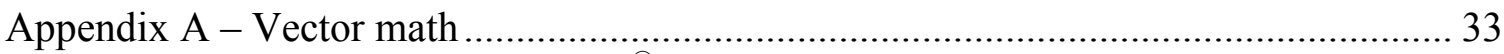

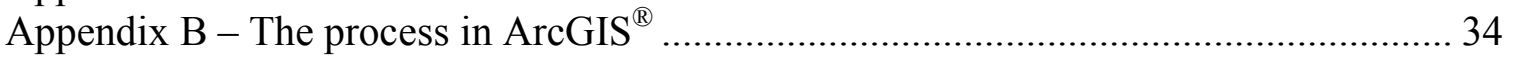

\section{Table of Figures}

Figure 1. Conceptual representation of the gnomonic projection..................................... 1

Figure 2. Aspects of the gnomonic projection........................................................... 2

Figure 3. The central cylindrical projection............................................................ 3

Figure 4. The Mercator projection...................................................................... 4

Figure 5. The plate carrée projection. ...................................................................... 4

Figure 6. The cylindrical equal-area projection........................................................... 5

Figure 7. The Gall orthographic projection. ......................................................... 5

Figure 8. The uninterrupted sinusoidal projection.......................................................... 6

Figure 9. The uninterrupted Mollweide (homolographic) projection................................ 7

Figure 10. The Robinson projection. ……………….............................................. 7

Figure 11. Goode's interrupted homolosine................................................................ 8

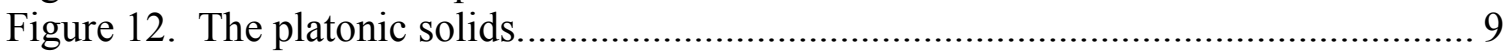

Figure 13. An icosidodecahedron, one of the 13 Archimedean solids. ......................... 10

Figure 14. Elongated triangular pyramid, a regular heptahedron. .................................. 10

Figure 15. The parabigyrate rhombicosidodecahedron, one of the 92 Johnson solids.... 10

Figure 16. The gnomonic version of Cahill's map....................................................... 11

Figure 17. Snyder's equal-area tetrahedron.............................................................. 11

Figure 18. Snyder's equal-area hexagon (cube) ...................................................... 12

Figure 19. Snyder's equal-area octahedron............................................................. 12

Figure 20. Snyder's equal-area dodecahedron. ........................................................... 13

Figure 21. Snyder's equal-area icosahedron. ............................................................... 13

Figure 22. World map on a thirty-two faced icosidodecahedron..................................... 14

Figure 23. World map on a regular heptahedron. ........................................................... 14 
Figure 24. World map on a parabigyrate rhombicosidodecahedron............................ 15

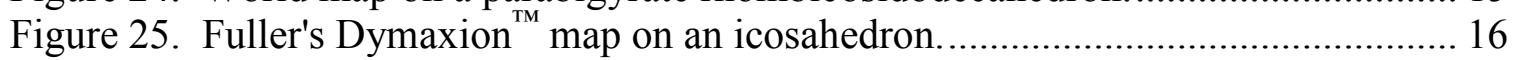

Figure 26. Tissot's indicatrix applied to one face using Fuller's projection (left) and the

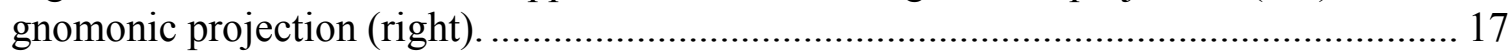

Figure 27. Fuller (shown in gray) and gnomonic (shown in orange). .......................... 17

Figure 28. Irregular heptahedron, an irregular seven-faced polyhedron. ....................... 18

Figure 29. Set of generating points in a plane (left) and the planar Voronoi diagram

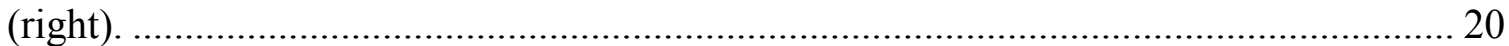

Figure 30. Set of generating points on a sphere (left) and the spherical Voronoi diagram

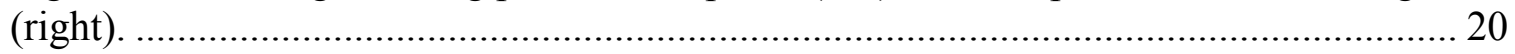

Figure 31. The right-hand rule, showing the effects of order on the vectors................. 21

Figure 32. Spherical Voronoi diagram and one of its "flattened" polygons.................... 23

Figure 33: Irregular heptahedron projection with Voronoi polygon boundaries ............. 24

Figure 34. Irregular heptahedron projection. ............................................................ 24

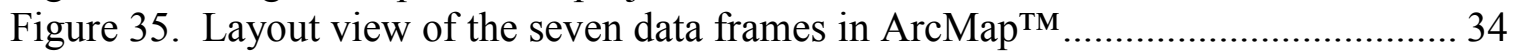

\section{List of Tables}

Table 1: The generating points (projective centers) 


\section{Introduction}

Displaying a portion of the Earth's curved surface on a flat surface such as a piece of paper or a computer screen requires the use of a mathematical transformation known as a map projection. It is impossible to create an exact planar representation of a spherical surface such as the Earth (American Cartographic Association [ACA], 1986; Fisher \& Miller, 1944). J. Benítez (2005) recently presented a simple mathematical proof that "a length preserving projection of the Earth is impossible" (p. 944). No map projection can preserve angles, areas, and distances. Some projections preserve one of these properties; others preserve two, but not throughout the entire map. Today, there are many map projections available to depict part of the Earth's surface on a flat medium. Many of these projections are meant to represent a single, specific portion of the Earth, and are not appropriate representations of the Earth as a whole. Many projections designed to map the Earth as a whole are plagued by excessive distortion in parts of the map.

This paper proposes a new method for creating polyhedral projections, using irregular polyhedra. Irregular polyhedra can provide more options for the placement of the interruptions within a polyhedron map. This can enhance readability while minimizing distortion. This method also reduces distortion by allowing the projective centers to be specified explicitly rather than depending on the predetermined arrangement provided by the geometry of a regular or semi-regular polyhedron. The method presented here uses irregular polyhedra based on gnomonically projected Voronoi partitions of the sphere.

\subsection{A perspective projection of the globe onto a plane}

One simple method of projecting the globe onto a plane is by the gnomonic projection (also called the central or radial projection). The gnomonic projection can be envisioned as the result of a point light source at the center of the Earth that projects the Earth's surface features onto a tangent plane (Figure 1).

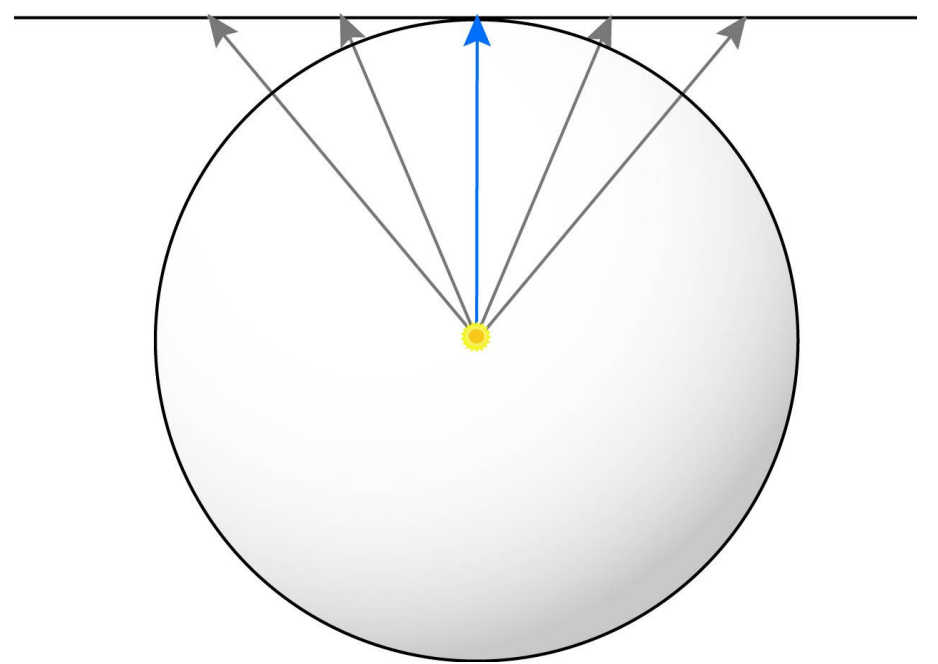

Figure 1. Conceptual representation of the gnomonic projection. 
On the gnomonic projection, the point of tangency is the only point without distortion, and distortion increases quickly away from this point (Snyder, 1987). Figure 2 shows different aspects of the gnomonic projection.

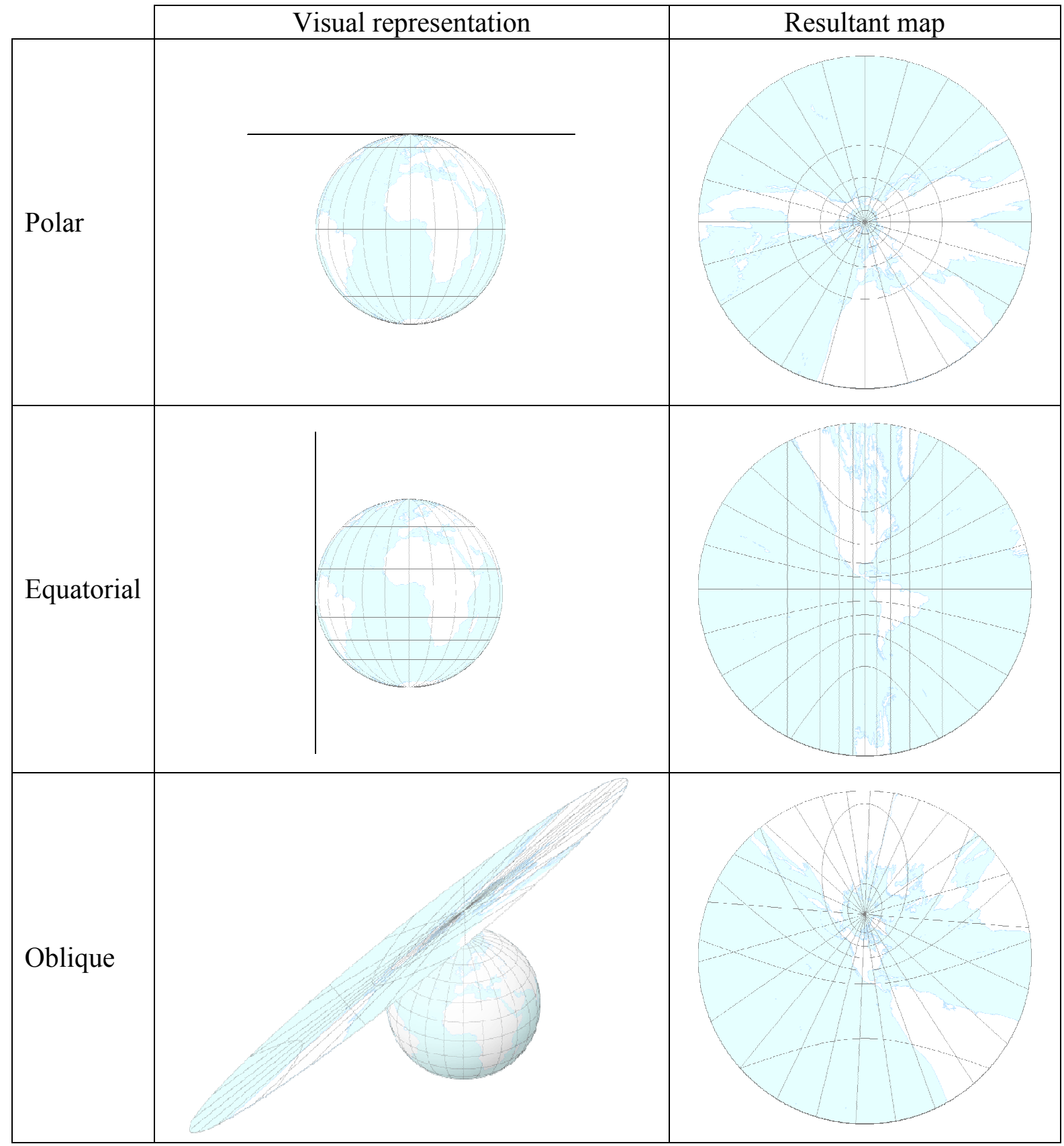

Figure 2. Aspects of the gnomonic projection.

The software used in creating Figure 2 has a display range of $75^{\circ}$ of spherical arc from the center of the projection. This means the resultant circular map has a field of view of $150^{\circ}$. The gnomonic projection is capable of showing just short of a hemisphere $\left(180^{\circ}\right)$ but the distortion on such a map would make it unintelligible.

A single gnomonic projection cannot be used for a world map, but it does have a unique property that allows multiple gnomonic projections to be joined together to form a world 
map. The gnomonic projection has the unique property of depicting all great circle paths, the shortest distance between two points on a sphere, as straight lines (Snyder, 1987). This makes the gnomonic projection particularly well-suited for creating polyhedron maps because the face edges of the polyhedron are straight lines.

Theoretically, the gnomonic projection can be used to map the surface of a sphere to any convex polyhedron that surrounds the sphere. Many polyhedra have faces that are not all tangent to the same sphere. In these cases, a scale adjustment on the non-tangent faces is necessary to ensure the adjacent faces match along the edges.

\subsection{Uninterrupted projections used for world maps}

In addition to the plane, other developable surfaces include the cone and the cylinder. Many world maps use cylindrical projections. The cylindrical equivalent of the simple central projection onto the plane is the central cylindrical. Figure 3 shows the central cylindrical projection.

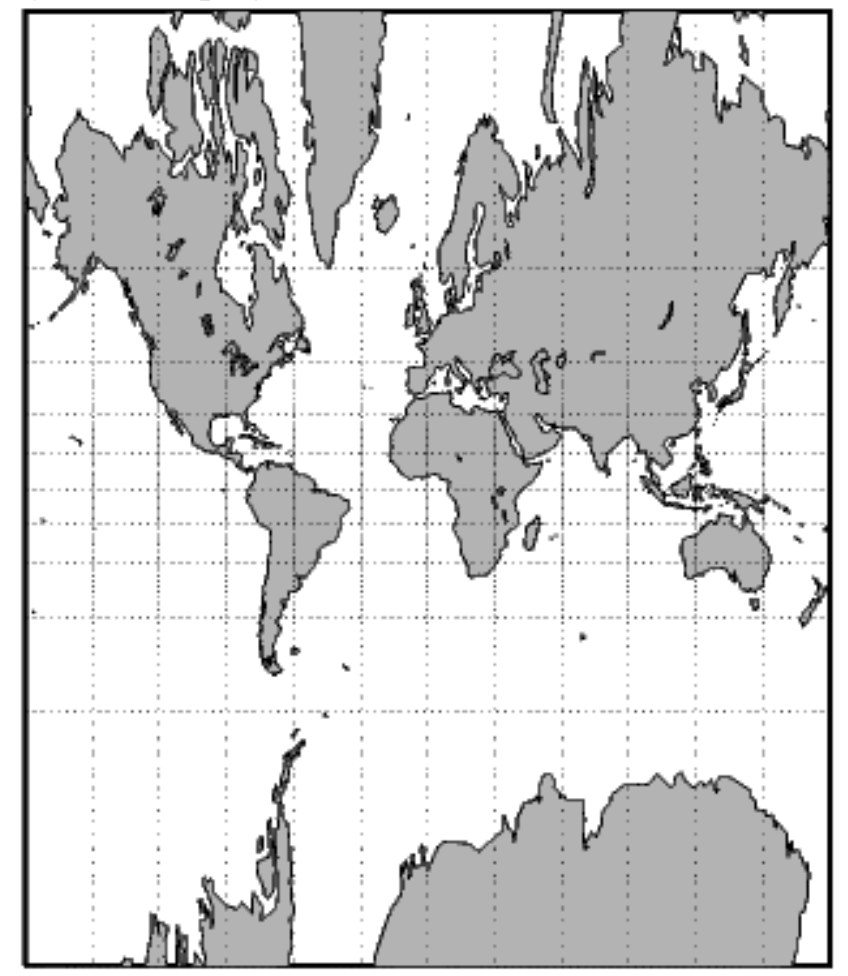

Figure 3. The central cylindrical projection (from http://www.mathworks.com/access/helpdesk_r13/help/toolbox/map/central.gif).

The central cylindrical can be envisioned as the result of a point light source at the center of the Earth that projects the Earth's surface features onto a tangent cylinder. In Figure 3, the cylinder is tangent at the equator. The poles cannot be shown (Snyder, 1993).

Figure 4 shows the Mercator projection, which also cannot show the Earth's poles. 


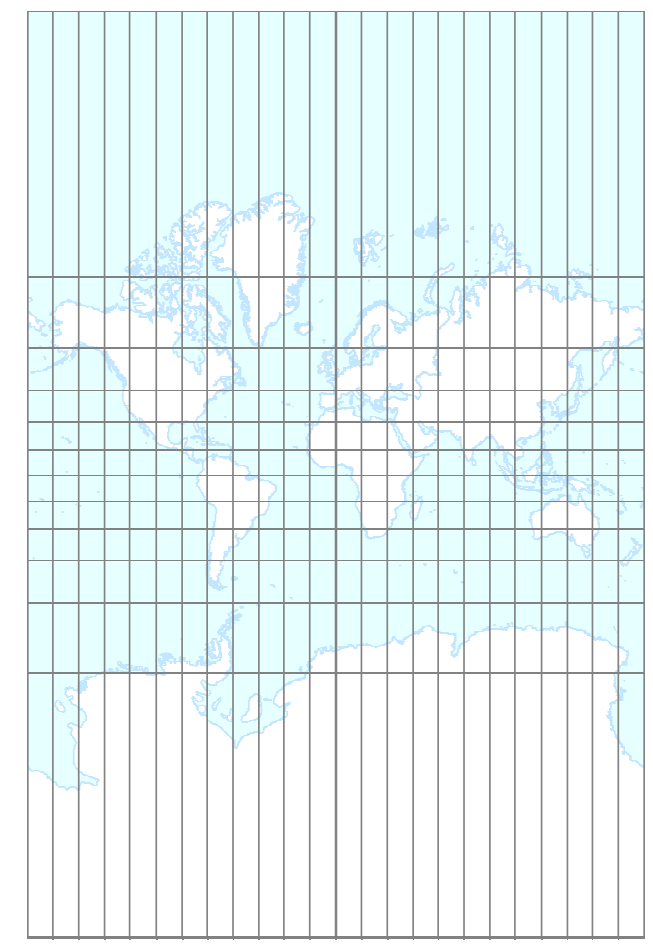

Figure 4. The Mercator projection.

In 1569, Gerardus Mercator presented this projection for use in navigation (Snyder, 1987). It is useful to navigators because lines of constant bearing (loxodromes or rhumb lines) appear as straight lines, making it easier to chart courses for ships. However, never intended for any use other than for navigation, it greatly distorts the land areas. Its misuse as a general world map in the first half of the 1900s sparked an interest in creating new world maps (Cahill, 1909).

Figure 5 shows the plate carrée projection, which is an equidistant cylindrical projection with the standard parallel at the equator.

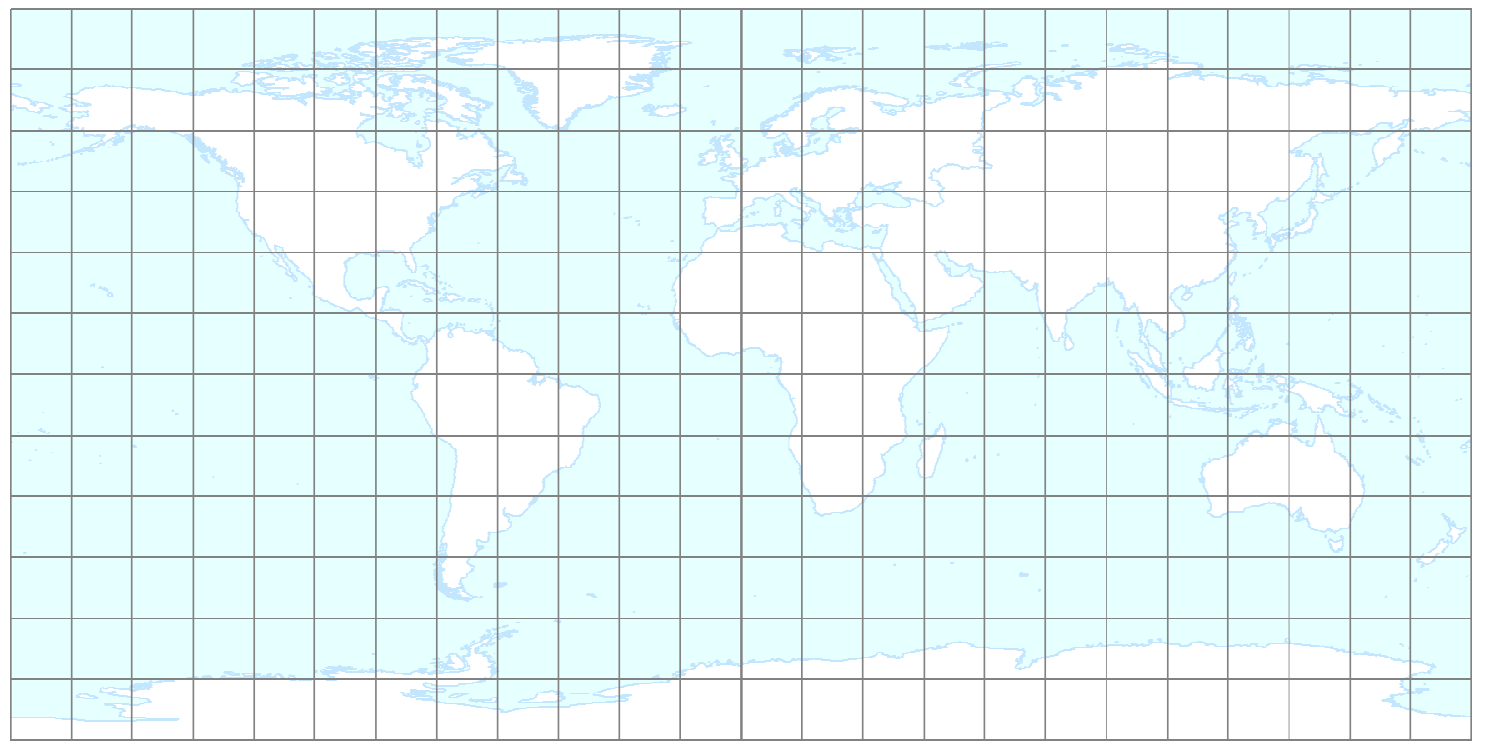

Figure 5. The plate carrée projection. 
The plate carrée projection is useful for showing latitudes near the equator and for correct scale along the meridians and the equator (Snyder, 1987), but has severe area and shape distortion elsewhere.

Figure 6 shows the cylindrical equal area projection.

\begin{tabular}{|c|c|c|c|c|c|c|c|c|c|c|c|c|c|c|c|c|c|c|c|c|c|c|c|}
\hline$=$ & 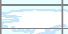 & 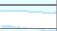 & $=$ & -9 & $+\infty$ & 5 & 5 & 8 & $\begin{array}{ll} \\
\end{array}$ & $=1$ & $=$ & 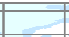 & $\overline{E F}$ & $-\infty$ & 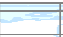 & 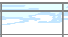 & $E-1$ & 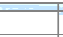 & 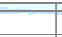 & 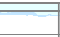 & $E$ & 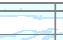 & $\Rightarrow$ \\
\hline & & & -4 & & & 3 & $=$ & 8 & & & of & 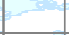 & & & 5 & & & & & & 5 & 5 & \\
\hline & & & & & & & & & & & $5=$ & $\frac{3}{3}$ & 5 & 7 & & & & & & & 3 & & \\
\hline & 40 & & & 4. & & & & & & & & & & & $\infty$ & 4 & & & $\gamma$ & & & & \\
\hline & & & & & & 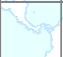 & 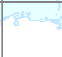 & & & & & $\operatorname{mon}$ & & & 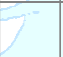 & & 6 & & 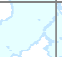 & 8 & & & \\
\hline & & & & & & & & & & & & & & & & & & & & & & $2 \times$ & \\
\hline & & & & & & & & & & & & & & & & & & & & & & & \\
\hline & & & & & & & & & & & & & & & & & & & & & 4 & & 3 \\
\hline & & & & & & & $c^{5}$ & & & & & & & & & $=$ & & & & & & & \\
\hline & & & & & & & 5 & & & & - & $=$ & & $\bar{L}$ & & 2 & & & & & & & $=$ \\
\hline
\end{tabular}

Figure 6. The cylindrical equal-area projection.

Presented by Johann Heinrich Lambert in 1772, this projection is an equal-area (equivalent) projection, meaning that all areas are equivalent to the areas on a globe of the same scale. However, there is severe shape distortion away from the equatorial latitudes, making its use as a world map difficult to justify.

Figure 7 shows the Gall orthographic (sometimes called Gall-Peters) projection.

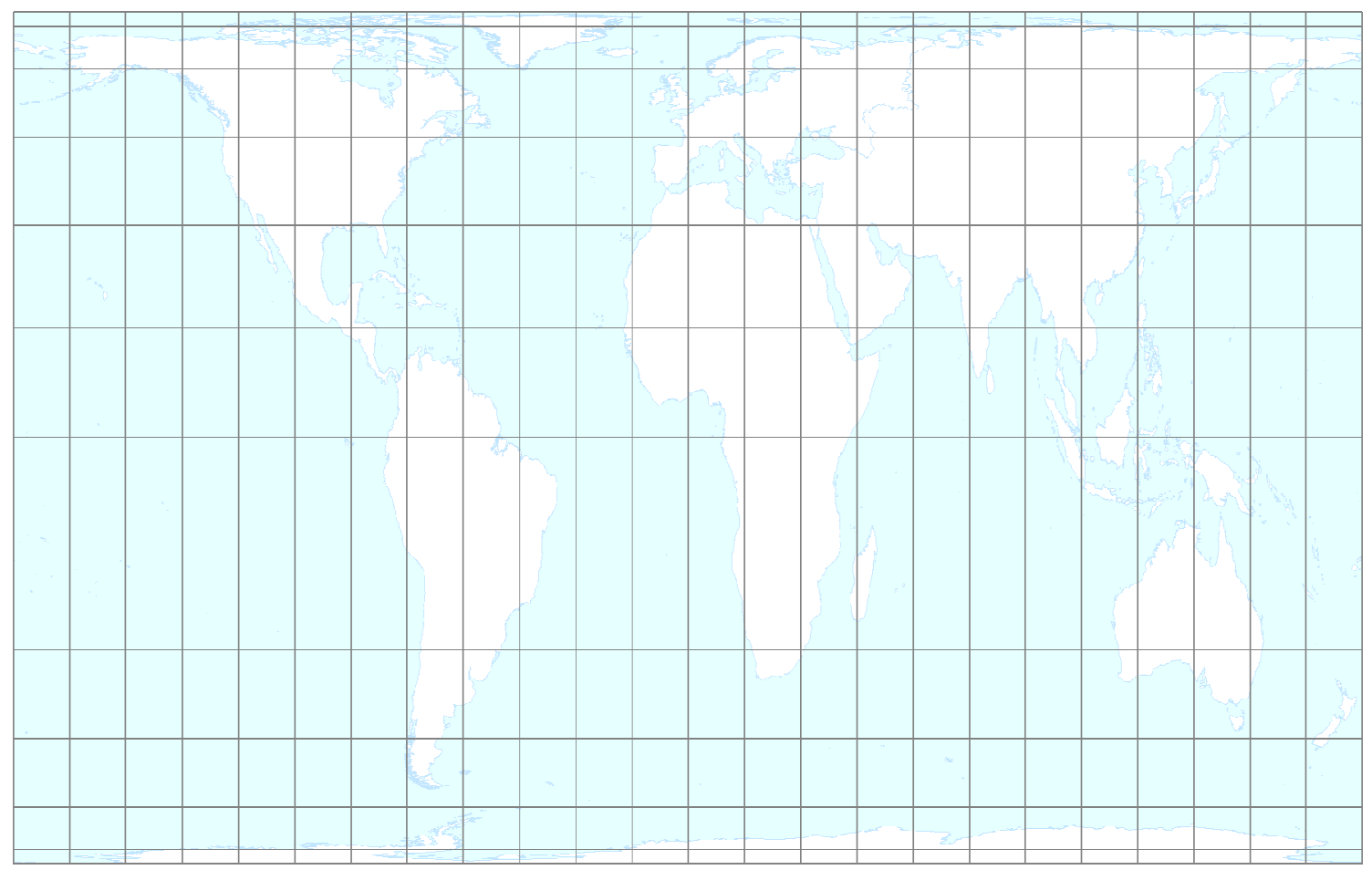

Figure 7. The Gall orthographic projection.

Presented by James Gall in 1855, the Gall orthographic projection is similar to the cylindrical equal-area projection but with standard parallels set to $45^{\circ} \mathrm{N}$ and $45^{\circ} \mathrm{S}$ 
(Snyder, 1987). This helps reduce some of the shape distortion found on the cylindrical equal-area projection, but it increases the shape distortion in the equatorial latitudes.

Figure 8 shows the uninterrupted sinusoidal projection.

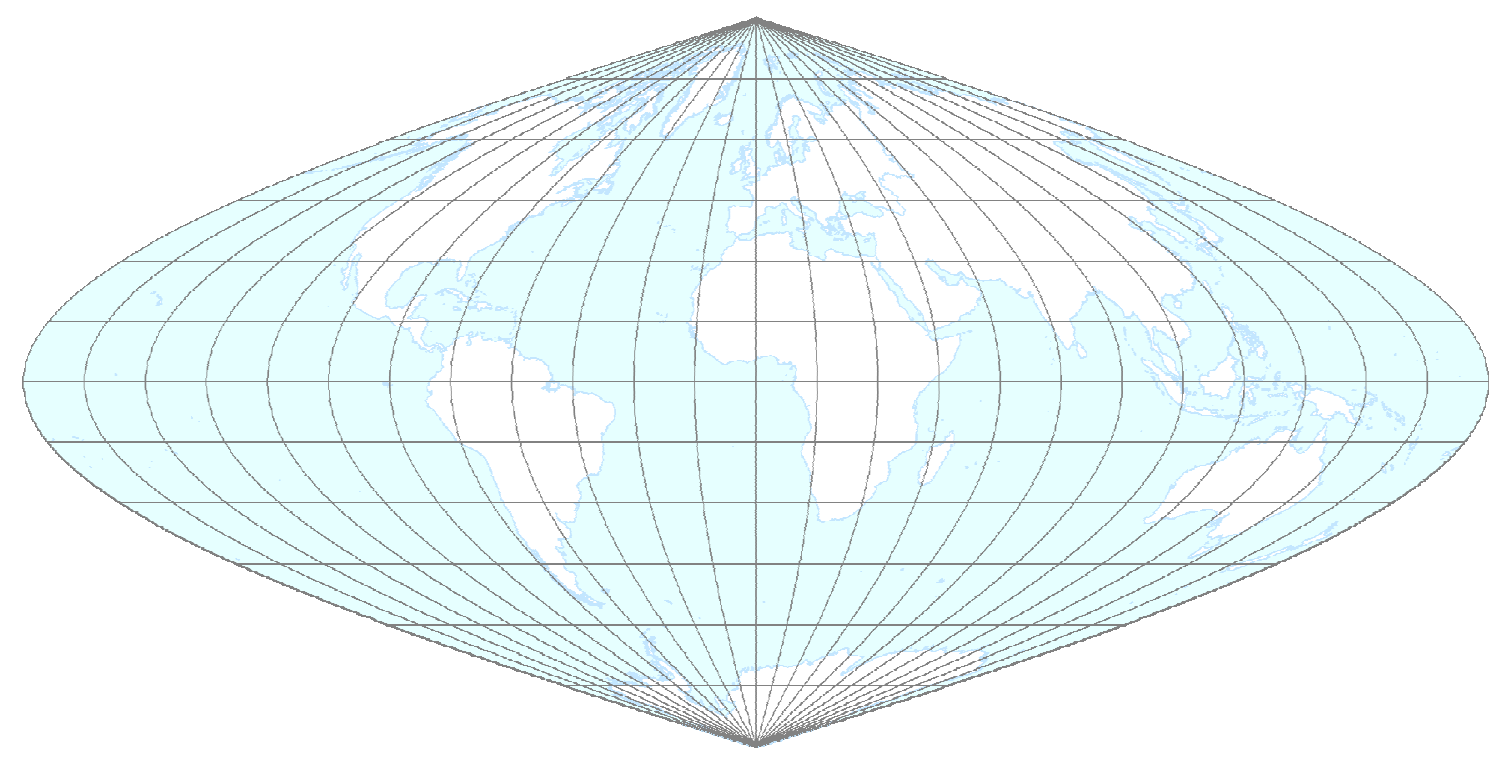

Figure 8. The uninterrupted sinusoidal projection.

In use since the mid- $16^{\text {th }}$ Century, the sinusoidal projection is the oldest extant pseudocylindrical (parallels and central meridian are straight lines, all other meridians are curves) projection (Snyder, 1987). The sinusoidal preserves area, but severely distorts shape. Its central meridian is shown as a straight line of true scale while all other meridians are shown as sinusoidal curves. The parallels are shown as parallel, equally spaced straight lines of true scale. Its primary uses are for world maps and for maps of South America and Africa (Snyder, 1987).

Figure 9 shows the uninterrupted Mollweide projection. 


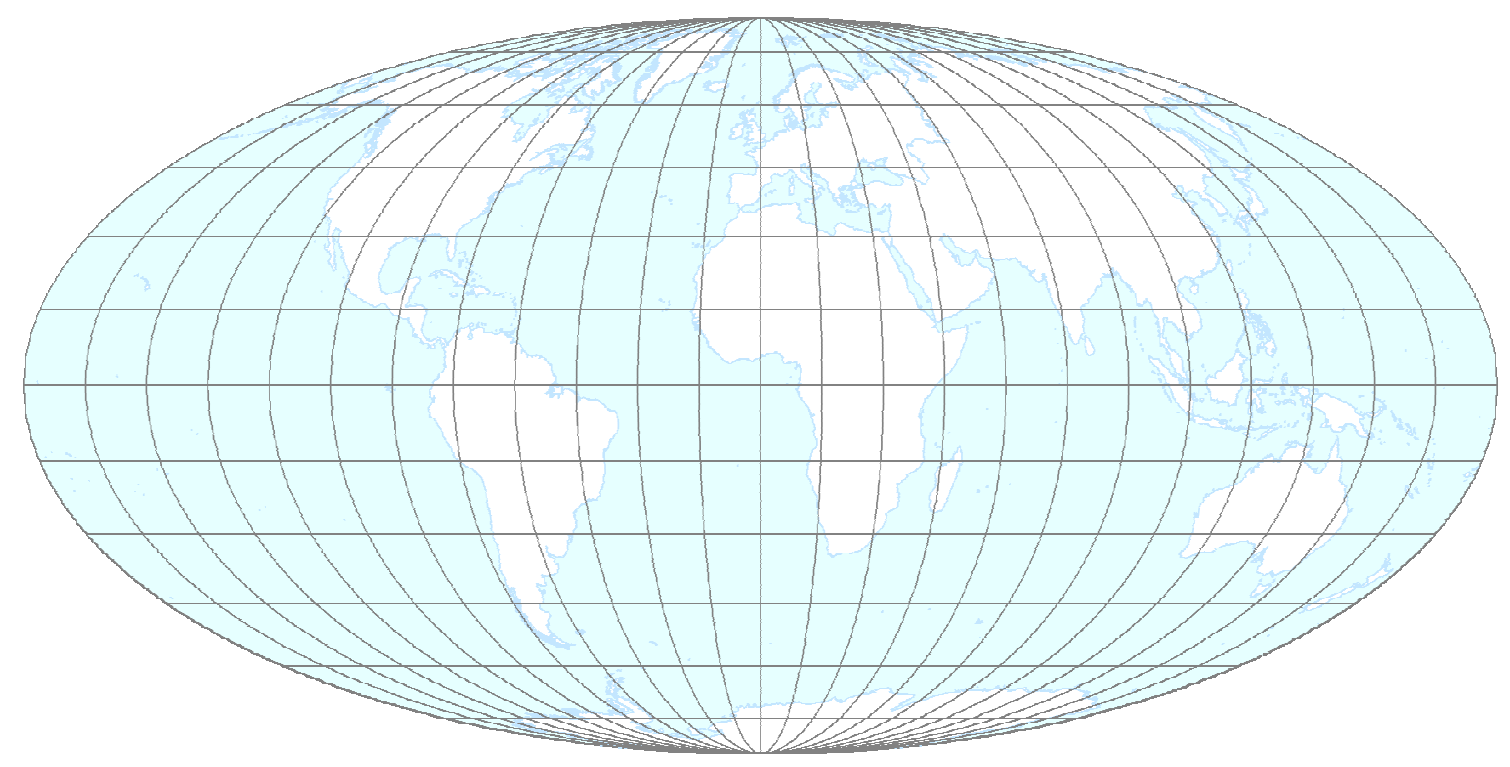

Figure 9. The uninterrupted Mollweide (homolographic) projection.

The Mollweide, created by Carl B. Mollweide in 1805, is considered to be the second oldest pseudocylindrical projection. Also known as the homolographic, the Mollweide is an equal-area projection, but severely distorts shape throughout the map. Its central meridian is a straight line and the other meridians are shown as elliptical curves, with the $90^{\text {th }}$ meridians forming a circle. Its parallels are parallel, unequally spaced straight lines (Snyder, 1987).

Figure 10 shows the Robinson projection, presented by Arthur H. Robinson in 1913.

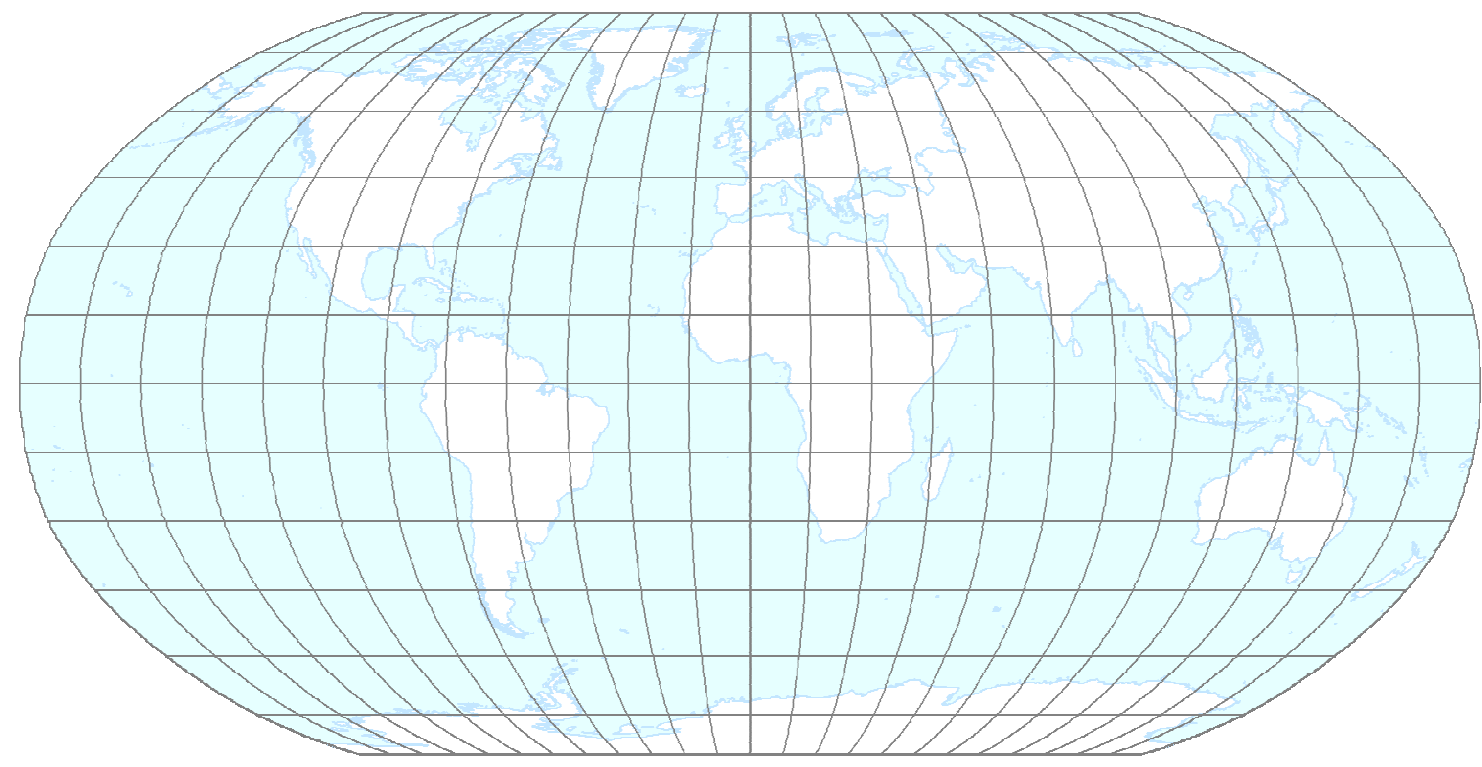

Figure 10. The Robinson projection.

The Robinson projection is a compromise projection (ACA, 1986). It does not preserve angles, areas, or distances, but attempts to lessen the distortion of all three properties. 


\subsection{Interrupted projections used for world maps}

Interrupting a map can reduce distortion (ACA, 1988). An interrupted map is achieved by joining multiple projections of the same or different types with different central meridians or projective centers. The purpose is to reduce distortion that is typically more pronounced toward the edges of the uninterrupted map.

\subsubsection{Goode's interrupted homolosine}

In 1916, pleased with the appearance of Africa on the uninterrupted sinusoidal, John Paul Goode developed an interrupted form of the sinusoidal with three central meridians, improving the appearance of South America, Southeast Asia, and Australia (Goode, 1925). This minimized the distortion effect created by the sinusoidal meridians as the distance increased from the central meridian (Snyder, 1987). More pleased with the way the uninterrupted Mollweide appeared toward the poles, he did the same with the Mollweide and then combined the best parts of both projections to create his interrupted homolosine (Figure 11), (Goode, 1925).

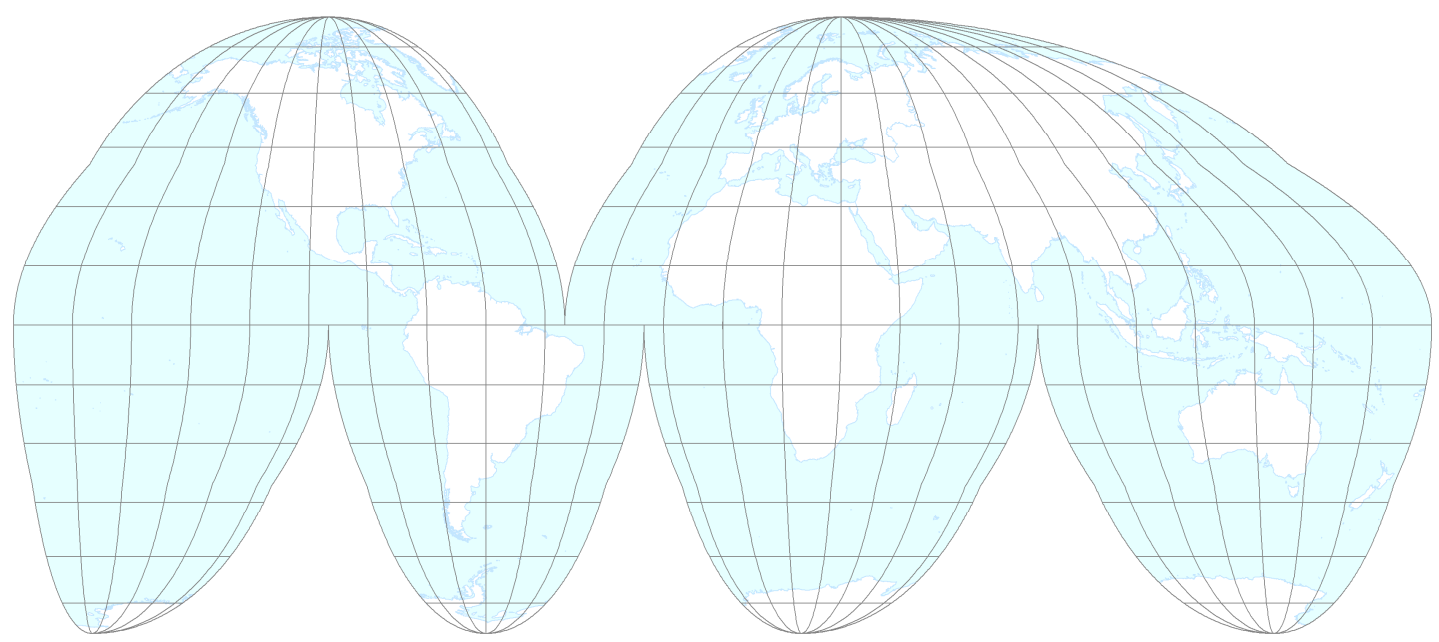

Figure 11. Goode's interrupted homolosine.

Lethcoe \& Klaver (1998) describe Goode's interrupted Homolosine as an "interrupted, pseudocylindrical, equal-area, composite map projection" (p. 1). Effectively, there are 12 projections that make up the homolosine: six Mollweide projections (two in the Northern hemisphere and four in the Southern hemisphere) with different central meridians, and six Sinusoidal projections (two in the Northern hemisphere and four in the Southern hemisphere) with different central meridians. When plotted at the same equatorial scale, the Mollweide and Sinusoidal projections match at one pair of latitudes: approximately $40^{\circ} 44^{\prime} 11.98^{\prime \prime} \mathrm{N}$ and $40^{\circ} 44^{\prime} 11.98^{\prime \prime} \mathrm{S}^{1}$ (Snyder, 1993). Hence, Goode joined the projections at these latitudes. This in turn created a visually detectable bend in the meridians, but still preserved the equal-area property.

\footnotetext{
${ }^{1}$ The actual value is $40^{\circ} 44^{\prime} 11.983883105076$ " when calculated on a sphere.
} 


\subsubsection{Polyhedral projections}

Another type of interrupted projection for world maps is a polyhedral projection. Polyhedral projections use polyhedra as the developable surfaces. Using polyhedra provides a means of joining multiple projections for controlling distortion.

A polyhedron is a closed surface or three-dimensional solid created from polygons joined along their edges. They can be convex or concave, but only convex polyhedra are explored in this paper. According to Eric Weisstein (2002), "a convex polyhedron can be defined as a polyhedron for which a line connecting any two (noncoplanar) points on the surface always lies in the interior of the polygon." Another way to describe a convex polyhedron is to say that it is bounded by convex polygons. Within the GIS discipline, this is similar to the idea of a convex hull.

In his article, "An equal-area map projection for polyhedral globes," (1992), Snyder describes the use of polyhedra for map projections:

Polyhedral globes have been used as approximations for spherical globes for centuries. The artist Albrecht Dürer (1538) first called attention to them, although he did not discuss map projections. Many innovators of the $19^{\text {th }}$ and $20^{\text {th }}$ centuries applied the Gnomonic projection to most of the common polyhedra at one time or another (Snyder \& Steward 1988). Folded polyhedral globes are easier to assemble without special techniques than spherical globes and serve as instructional tools, but they are bulky and small-scale, like globes. Unfolded and flattened polyhedral globes form world maps on projections which can have less distortion than other interrupted projections, but there are generally an increased number of interruptions and greater complications in plotting (p.10).

The types of polyhedra that have been commonly used for map projections are regular and semiregular polyhedra and, to a lesser extent, prisms and antiprisms. The five regular polyhedra, known as the Platonic solids, are the tetrahedron, hexahedron (cube), octahedron, dodecahedron, and icosahedron (Figure 12).
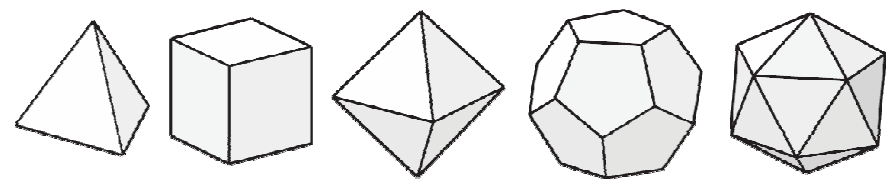

Figure 12. The platonic solids.

The Platonic solids have regular faces of the same type with the same number of faces sharing each vertex (Cromwell, 1997). Each Platonic solid also has a circumsphere, a sphere on which its vertices lie, and an insphere, an interior sphere which touches each face.

The semiregular polyhedra are also known as the Archimedean solids. The polyhedra in this group are highly symmetric arrangements of regular polygons of two or more different types. The edges of the polygons are all of the same length. The Archimedean solids have circumspheres but do not have inspheres (Weisstein, 2004, Insphere). There are 13 Archimedean solids with number of faces ranging from 8 to 92 (Weisstein, 2004, Archimedean Solid). Figure 13 shows the thirty-two faced icosidodecahedron. 


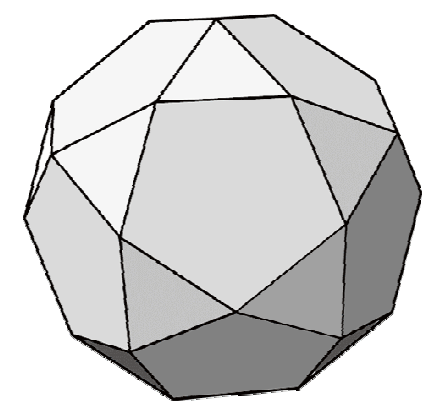

Figure 13. An icosidodecahedron, one of the 13 Archimedean solids.

Another family of regular-faced polyhedra is the Johnson solids. They are also arrangements of regular polygons of two or more different types but do not adhere to the strict symmetrical properties of the Archimedean solids. There are 92 Johnson solids with the number of faces ranging from 5 to 62 . Figure 14 shows an elongated triangular pyramid that uses three squares and four equilateral triangles. Different arrangements of the net, or unfolded polyhedron, are also shown.
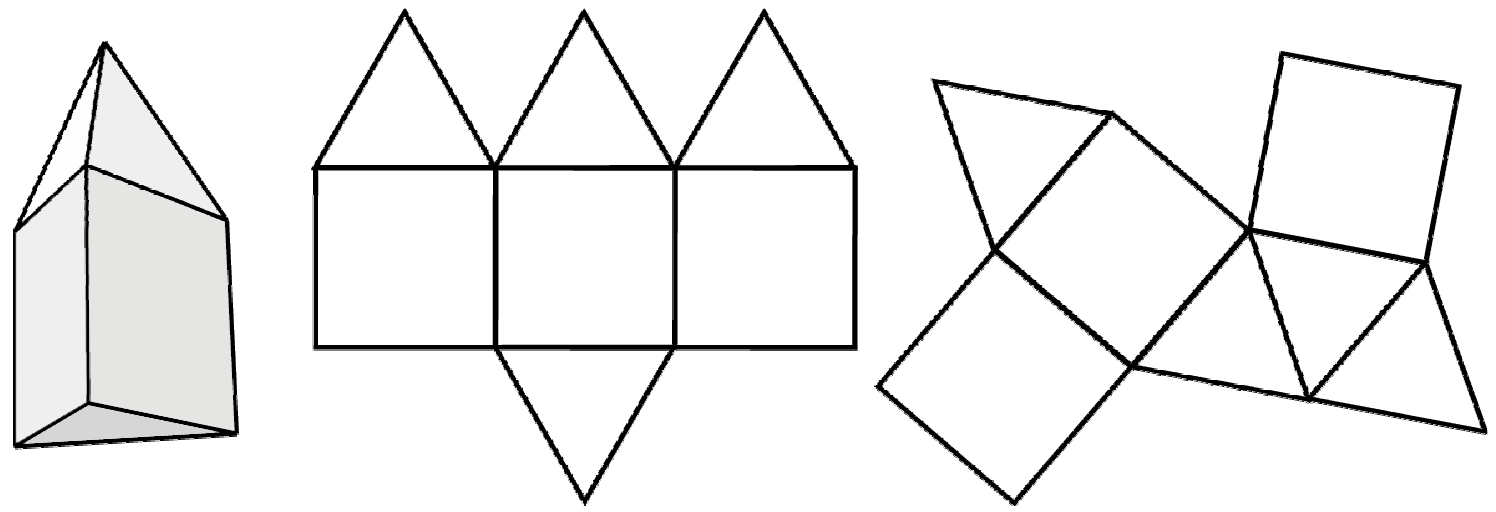

Figure 14. Elongated triangular pyramid, a regular heptahedron.

The Johnson solids can have many faces. Figure 15 shows the sixty-two faced parabigyrate rhombicosidodecahedron (Weisstein, 2006).

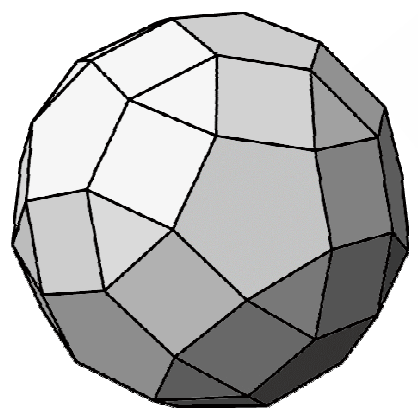

Figure 15. The parabigyrate rhombicosidodecahedron, one of the 92 Johnson solids.

Other types of polyhedra include the rhombic solids, which have rhomboids for faces, and the Catalan solids, which are the duals ${ }^{2}$ of the Archimedean solids. The Catalan

\footnotetext{
${ }^{2}$ A dual of a polyhedron takes its vertices to be at the face centers of the polyhedron.
} 
solids are constructed from polygons that can have different edge lengths but the faces are all similar polygons.

\subsubsection{Cahill's map on an octahedron}

In 1909, frustrated by the misuse of Mercator's map as a general world map, Cahill presented a world map on an octahedron. Figure 16 shows the gnomonic version of Cahill's map using the coordinates for the vertices as specified in his U.S. patent of 1913 (Cahill, 1913).

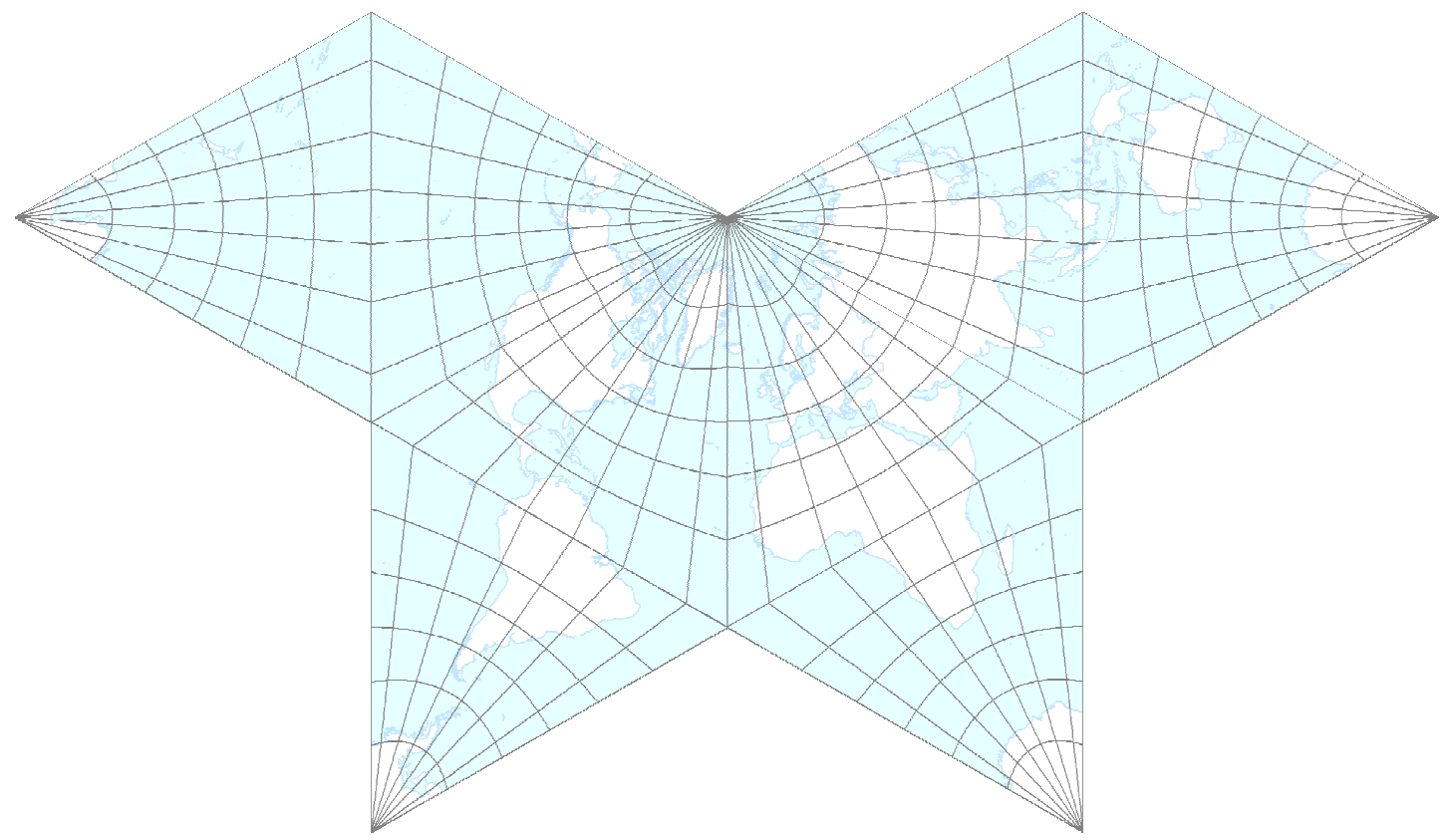

Figure 16. The gnomonic version of Cahill's map.

\subsubsection{Snyder's maps on polyhedra}

In 1992, Snyder presented equal-area maps on regular polyhedra. Figures 17-21 show his maps.

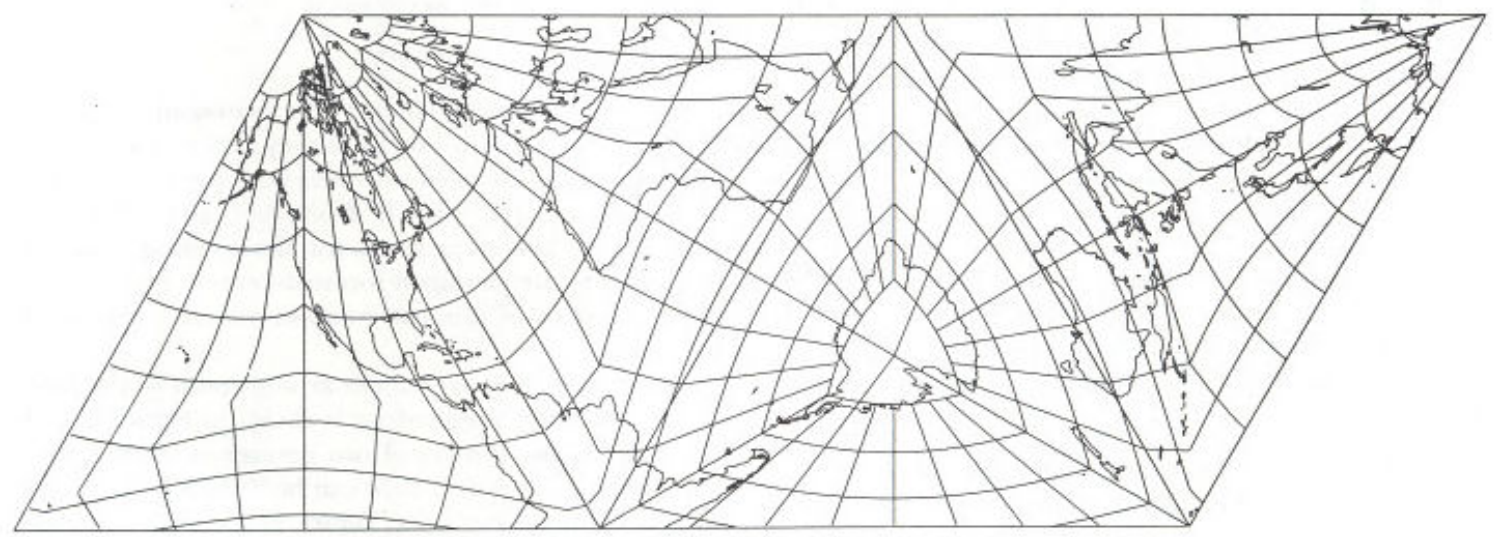

Figure 17. Snyder's equal-area tetrahedron (Snyder, 1992). 


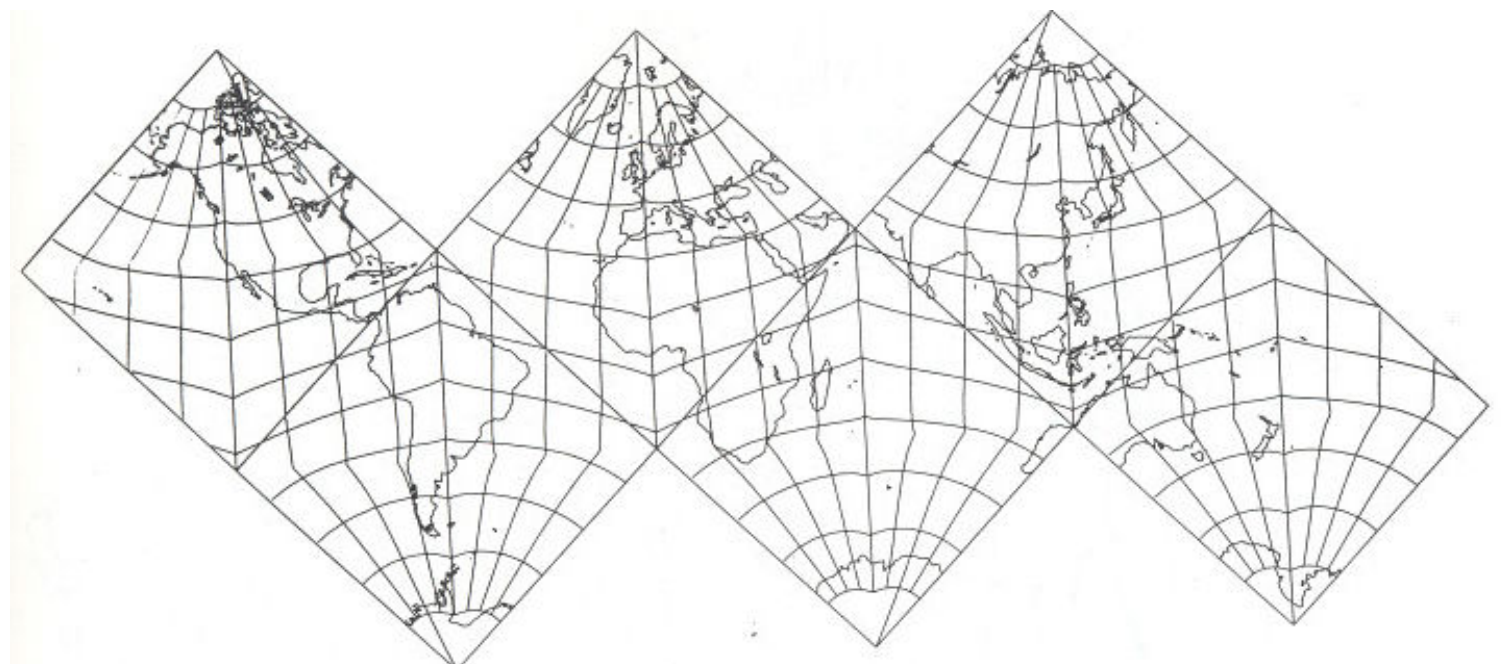

Figure 18. Snyder's equal-area hexagon (cube) (Snyder, 1992).

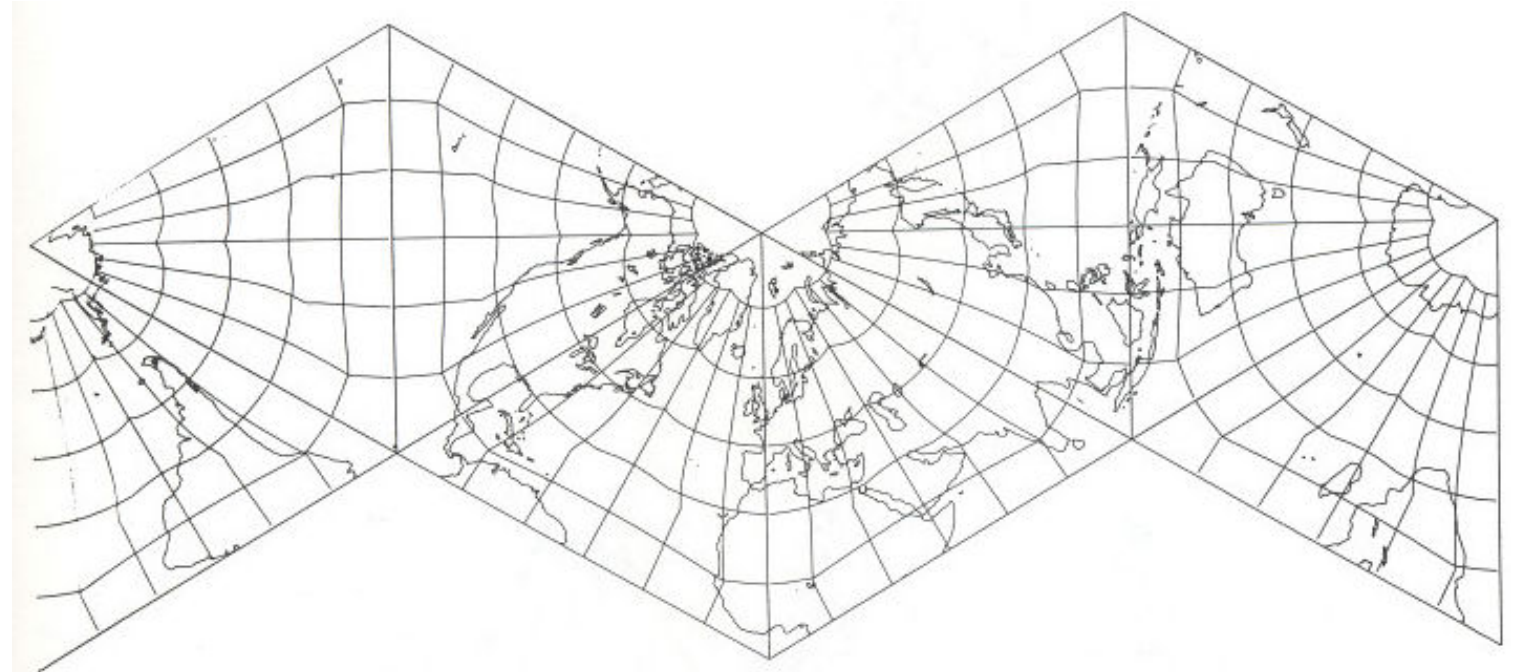

Figure 19. Snyder's equal-area octahedron (Snyder, 1992). 


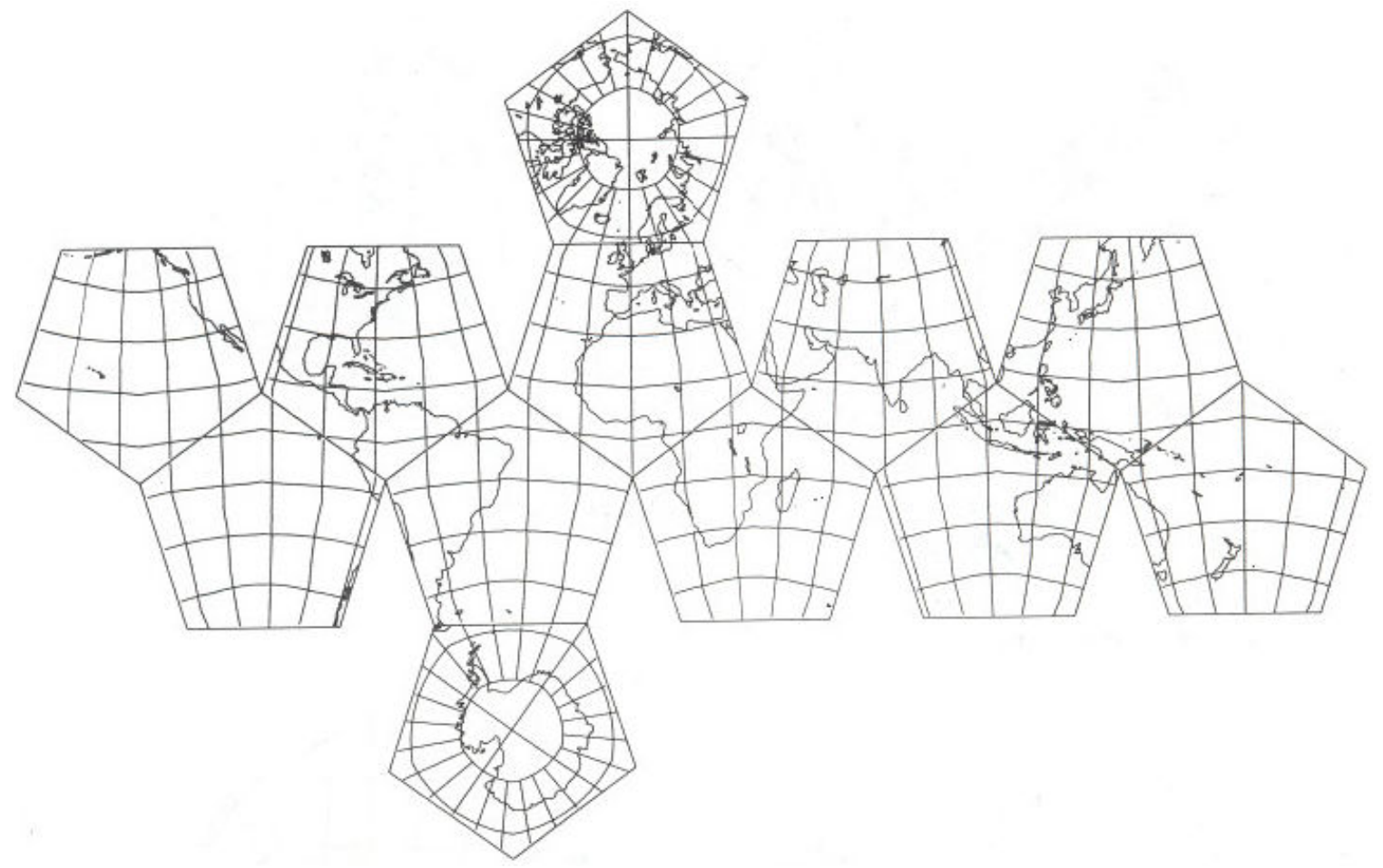

Figure 20. Snyder's equal-area dodecahedron (Snyder, 1992).

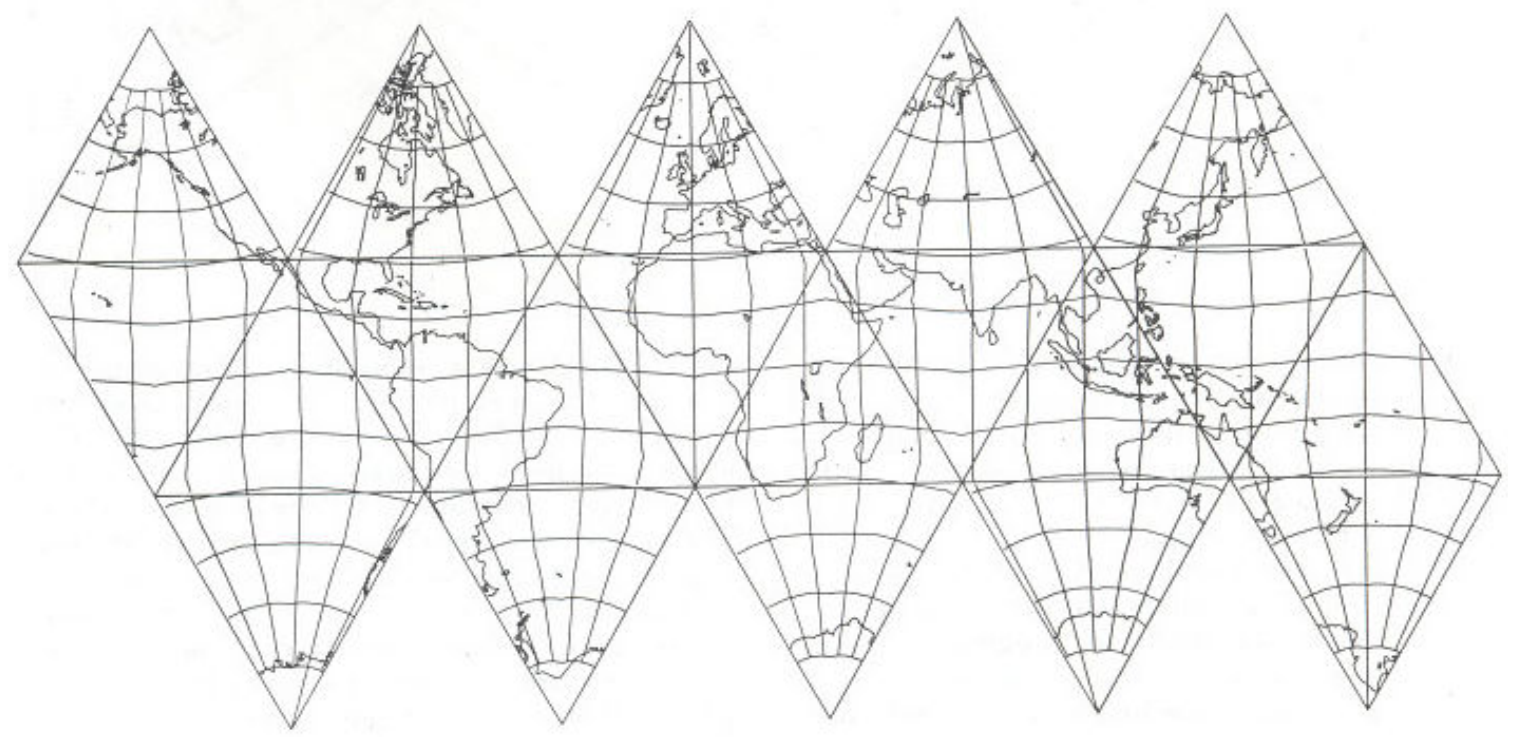

Figure 21. Snyder's equal-area icosahedron (Snyder, 1992).

\subsubsection{Hafner and Zitko's projections}

Izidor Hafner and Tomislav Zitko have produced many polyhedral world maps using the gnomonic projection. Figure 22 shows the world map on a thirty-two faced icosidodecahedron. 


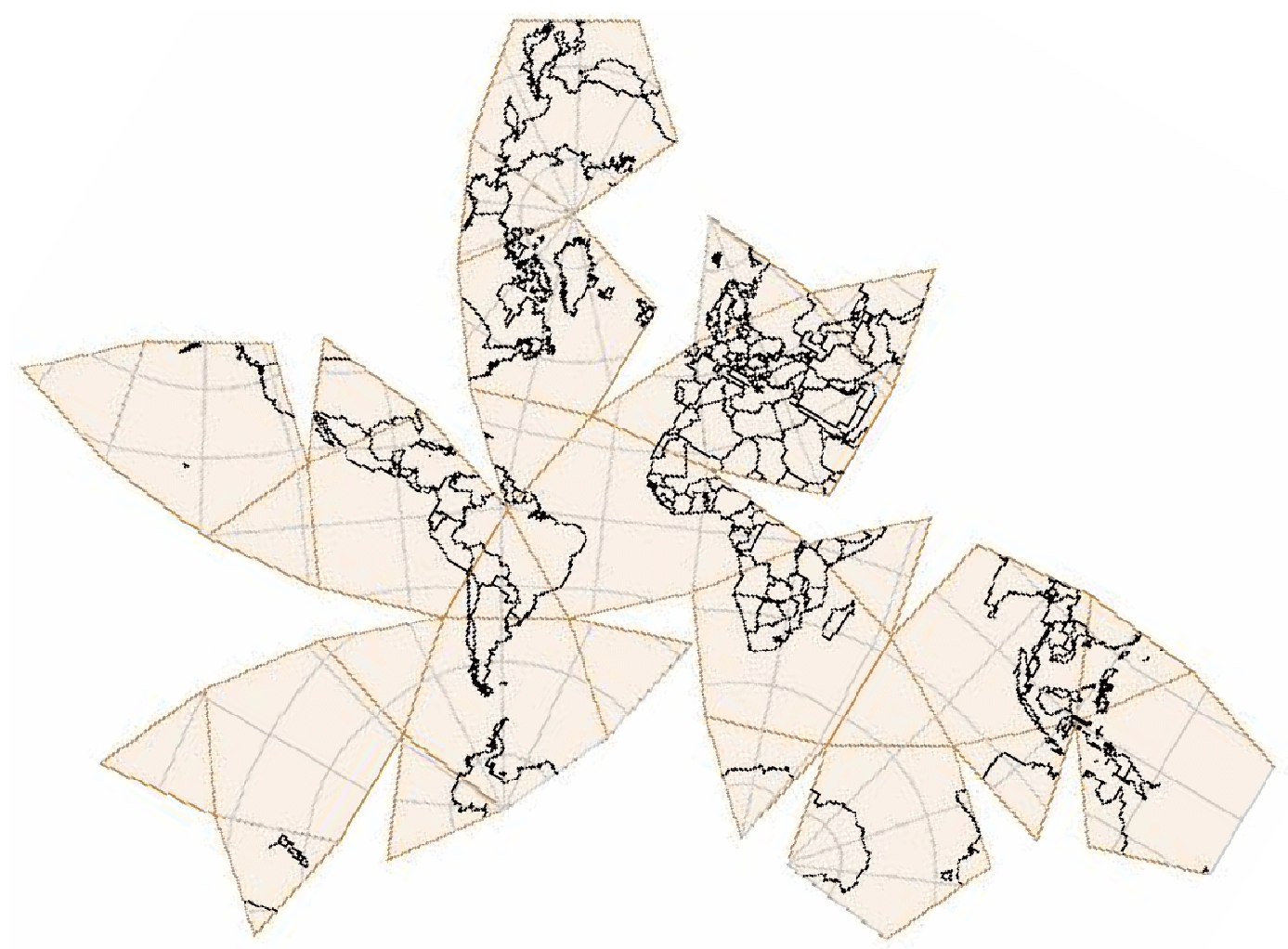

Figure 22. World map on a thirty-two faced icosidodecahedron (Hafner \& Zitko, n.d.).

Figure 23 shows a world map on a regular heptahedron.

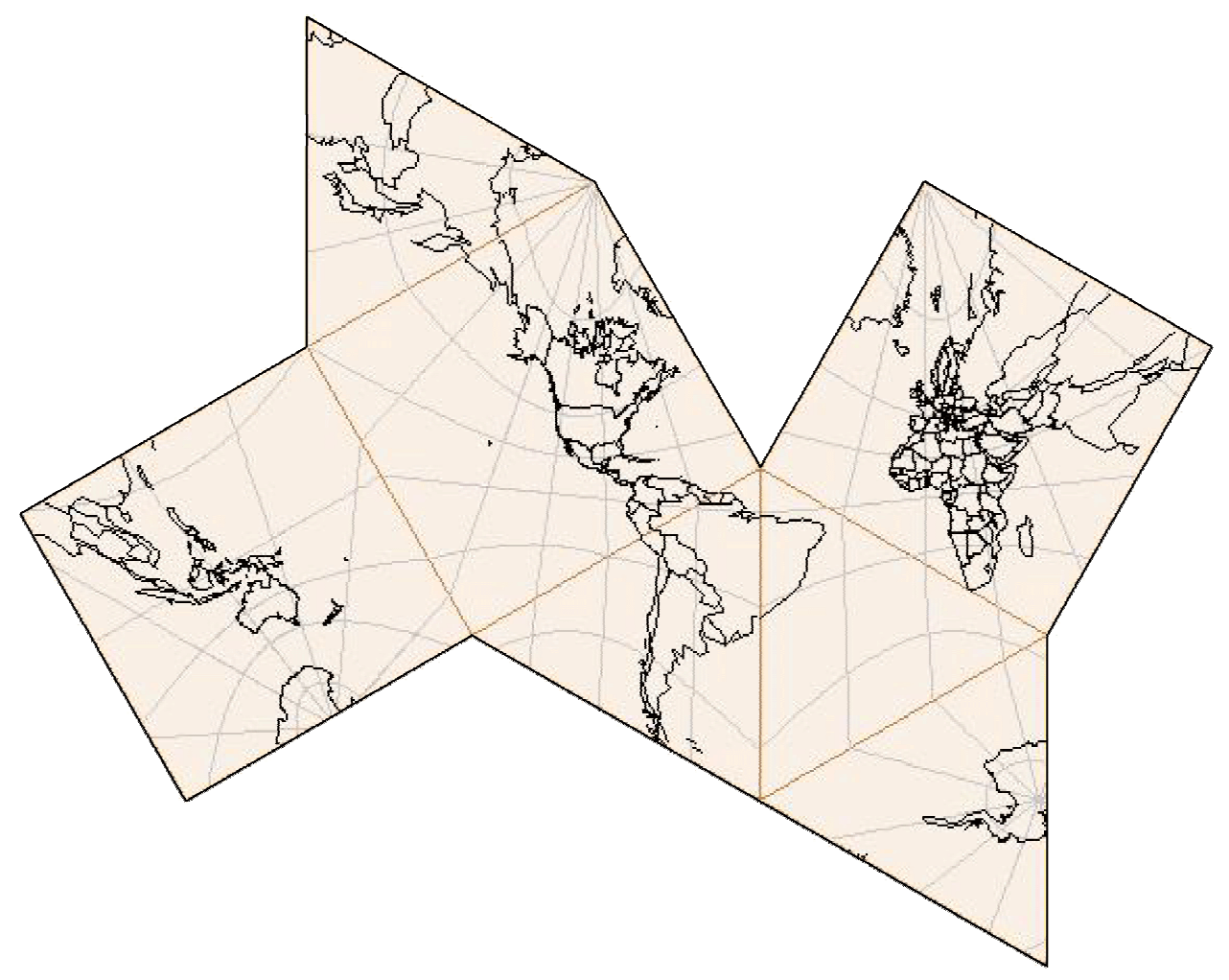

Figure 23. World map on a regular heptahedron (Hafner \& Zitko, n.d.). 
Figure 24 shows a world map on a parabigyrate rhombicosidodecahedron.

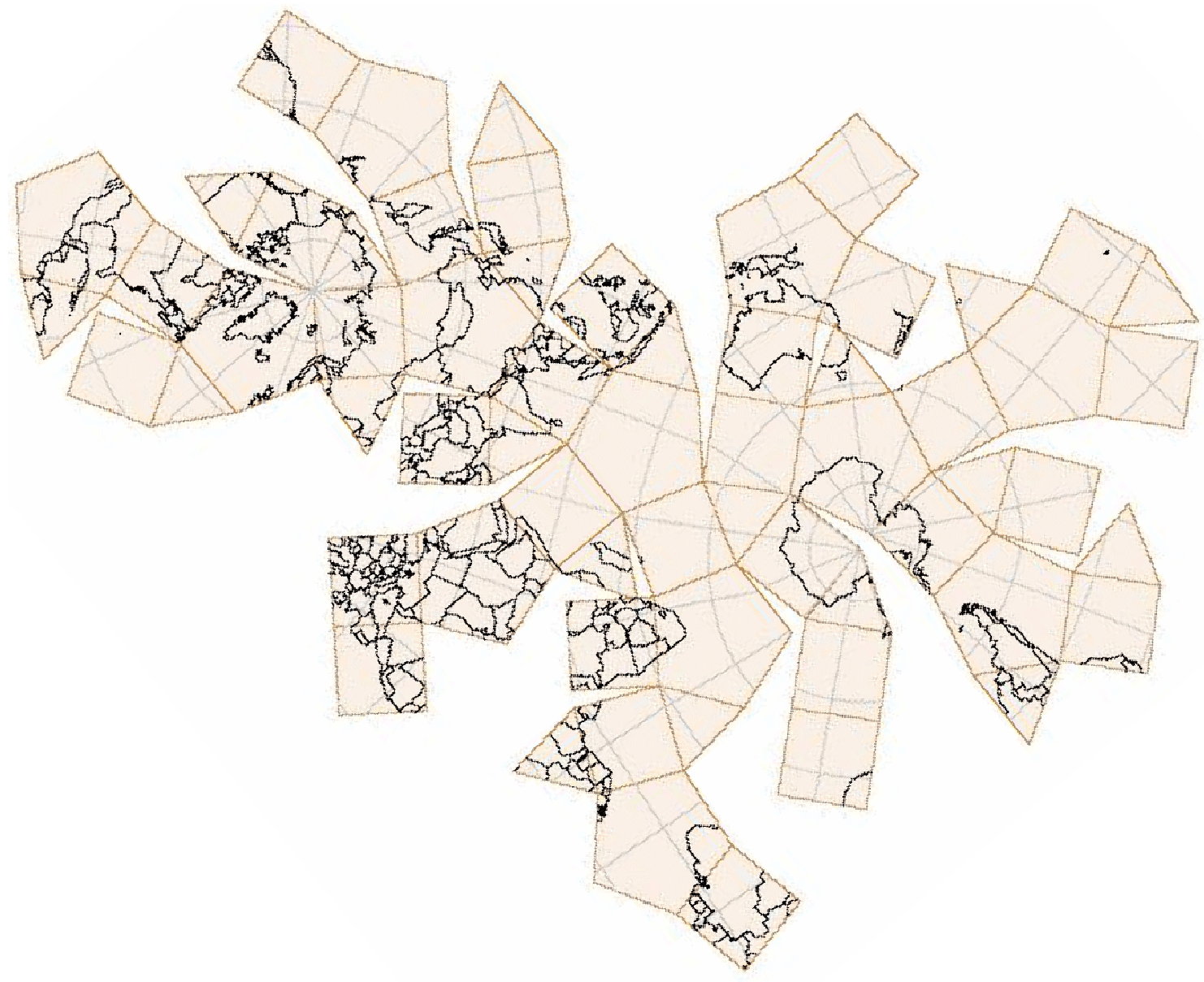

Figure 24. World map on a parabigyrate rhombicosidodecahedron (Hafner \& Zitko, n.d.). 


\subsubsection{Fuller's Dymaxion ${ }^{\mathrm{TM}}$ projection}

In 1954, Buckminster Fuller created the Dymaxion ${ }^{\mathrm{TM}}$ projection (Figure 25), using a regular icosahedron as its developable surface (Buckminster Fuller Institute, 2002).

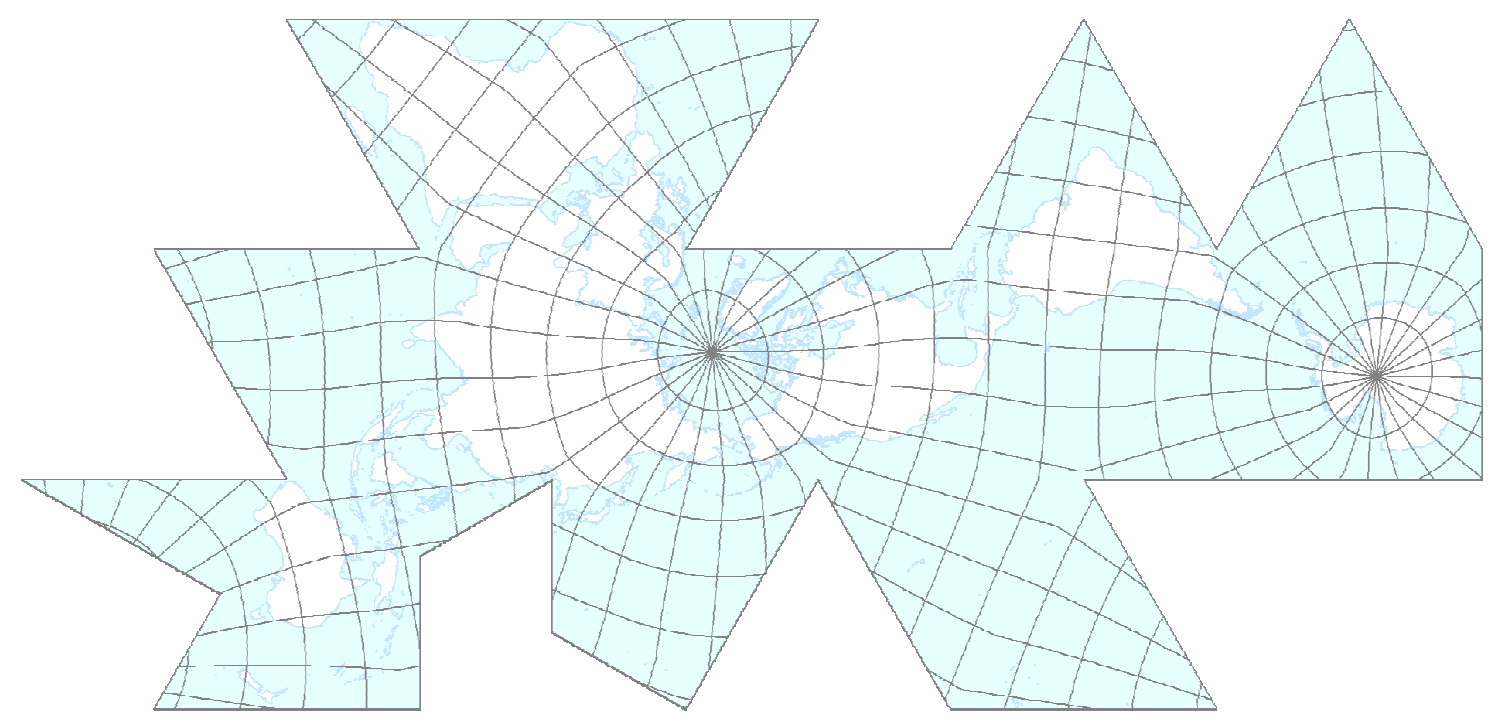

Figure 25. Fuller's Dymaxion ${ }^{\mathrm{TM}}$ map on an icosahedron.

A regular icosahedron approximates a sphere with 20 equilateral triangles. Fuller carefully positioned the icosahedron so that all of its vertices are in water areas. This allowed him to place the interruptions so that there are no breaks in the land masses when the 20 triangles are unfolded. Most of the triangles remain intact, but two had to be interrupted to preserve the continuity of the continents.

The transformation equations Fuller used were not published until 1995 by Robert Gray. Fuller had devised a new transformation that preserved length (scale) along the edges of each triangular face of the icosahedron. This allowed adjacent triangles to match along a shared edge. Since the surface area of a spherical triangle is greater than the area of a planar triangle with the same edge lengths, Fuller's transformation averaged out this extra area within the planar triangle. In the patent of an earlier, cuboctahedron version of his Dymaxion $^{\mathrm{TM}}$ map, Fuller (1946) wrote:

...it is possible to maintain uniform scale peripheral cartographic delineations and to distribute all subsidence distortion from the periphery toward the center. I have discovered further that this system brings the subsidence distortion to an irreducible minimum which, without correction of any kind, is very considerably less than with any system of projection heretofore devised (p. 1).

The greatest area distortion occurs towards the center of the triangle and the least amount of area distortion occurs towards the vertices (Gray, 1995). Visually, however, most of the shape distortion occurs at the vertices of the triangles. This result is similar to the gnomonic projection, in which distortion increases away from the projective center.

Tissot's indicatrix is a mechanism for illustrating area and angular distortion (Tissot, 1881, as cited in Snyder, 1987). On a perfect map, Tissot's indicatrix would consist of circles of the same size throughout the map, indicating no distortion of area or angle. 
Area distortion is illustrated by a change in circle size. Angular distortion is illustrated by an elongation of the semimajor axis (Figure 26).
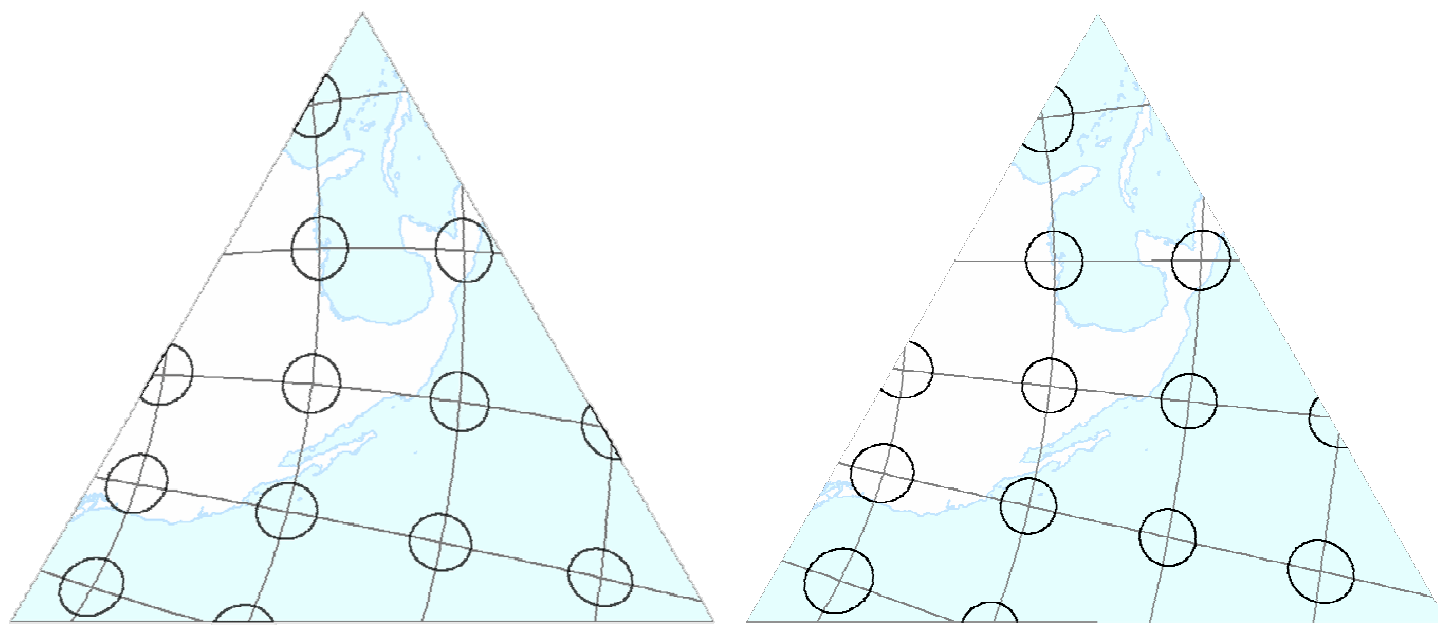

Figure 26. Tissot's indicatrix applied to one face using Fuller's projection (left) and the gnomonic projection (right).

The triangles in Figure 26 are not of the same nominal scale. The nominal scale for Fuller's transformation describes the scale at which the edges of the spherical triangles are the same length as the edges of the planar triangles. The nominal scale of the gnomonic projection describes the scale at the point of tangency, or the center of the triangle. At the same nominal scale, the gnomonic triangle would appear larger, due to the distortion at the vertices. Figure 26 shows the gnomonic triangle rescaled to the same size as Fuller's for ease of visual comparison.

Figure 27 shows the superimposed triangles to more easily detect the difference in shape distortion.

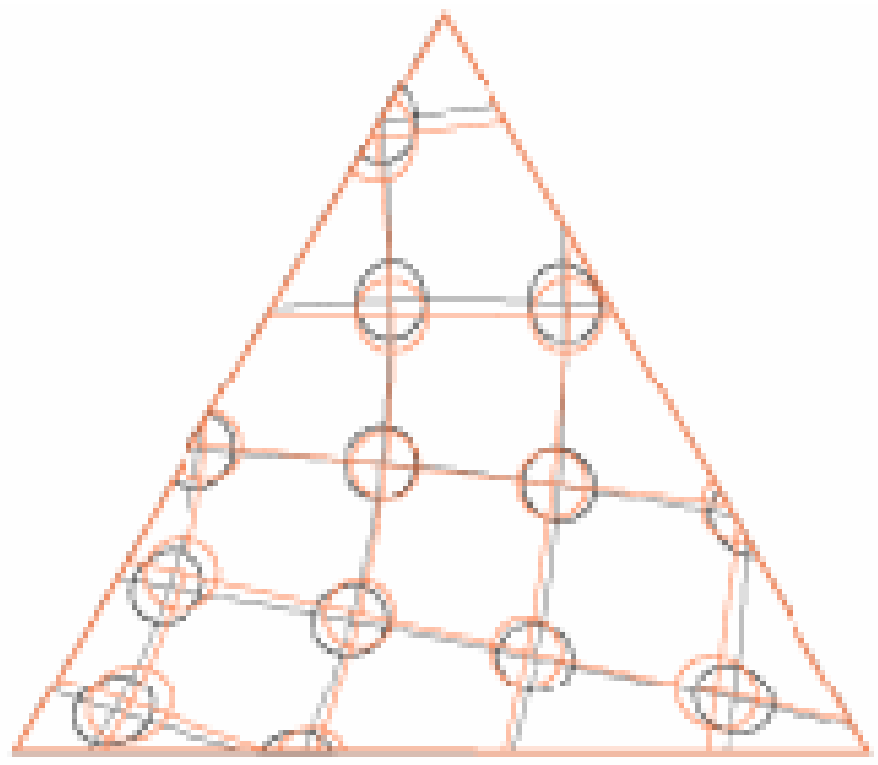

Figure 27. Fuller (shown in gray) and gnomonic (shown in orange). 
Fuller's transformation is different than a gnomonic projection onto an icosahedron, and shows only a slight difference in shape distortion.

\subsection{Irregular polyhedral projections}

Irregular polyhedral projections, an area left largely unexplored until now, may provide another means of creating low distortion world maps. A problem with regular and semiregular polyhedral maps is that they allow little control over the placement of the projective centers for each face. For a given polyhedron, the mapmaker is limited by its geometry for the placement of the projective centers. The method presented here creates a polyhedron tailored to specified areas of interest.

Irregular polyhedra are made of polygons that have different edge lengths (Figure 28).

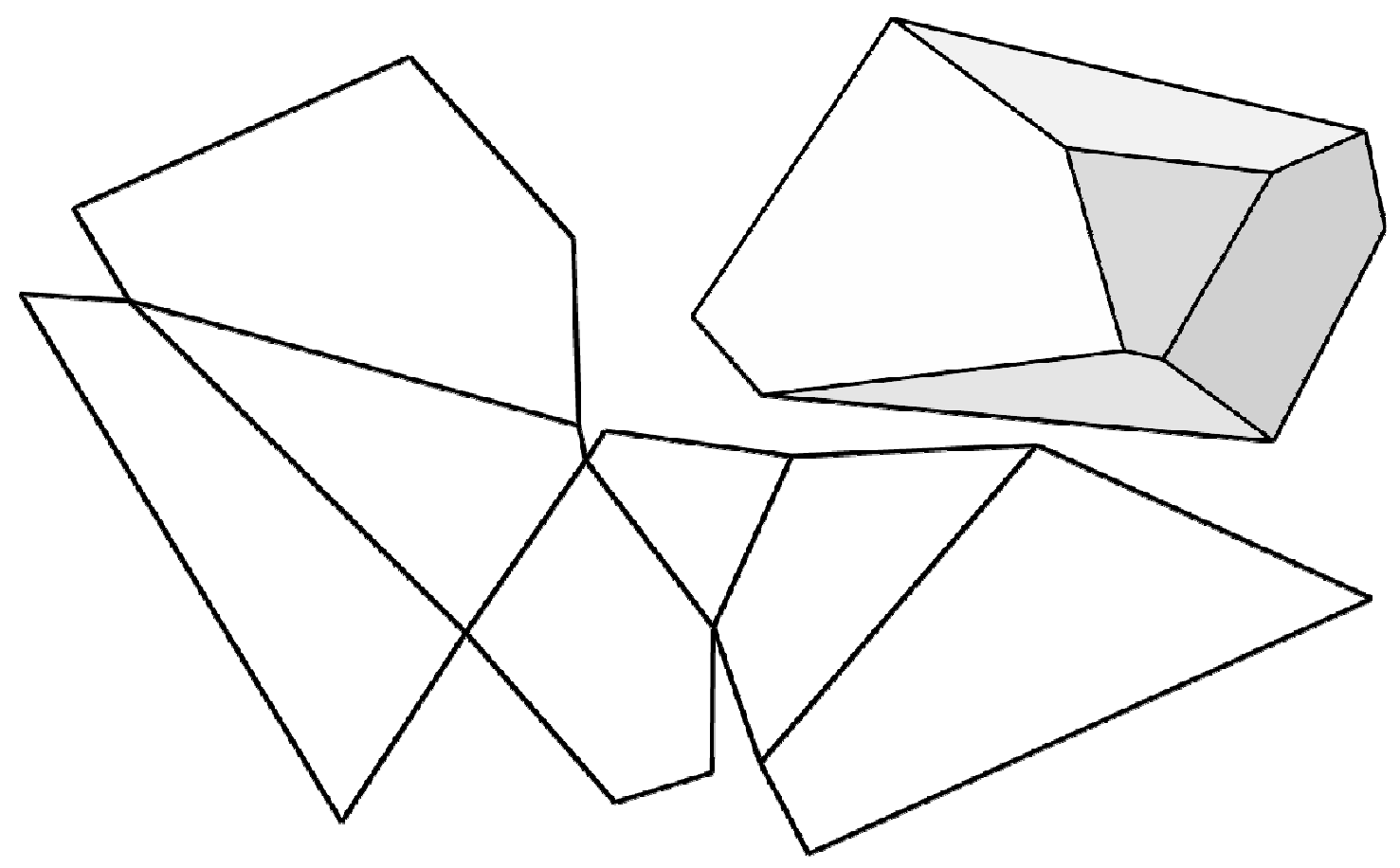

Figure 28. Irregular heptahedron, an irregular seven-faced polyhedron.

For the purpose of creating map projections, irregular polyhedra can provide a better fit to the land masses than the prearranged regular and semiregular polyhedra. In the regular and semiregular cases, the projective centers are determined by the polyhedra. This makes it difficult to control the distortion on the areas of interest within the map. In the method presented in this paper, the polyhedra and the projected centers are determined by the areas of interest. This is a better method for mapmaking since the Earth's land masses are not evenly distributed at regular intervals around a sphere. Also, a polyhedron map projection must be interrupted. Again, using regular and semiregular polyhedra limits the control of the placement of these interruptions as they are largely determined by the particular polyhedron chosen for the map. Another benefit of using an irregular polyhedron is that it provides more control when determining where a map will be interrupted. 


\section{Methodology}

The process presented here for creating an irregular polyhedron map projection involves creating a spherical Voronoi diagram and gnomonically projecting each spherical Voronoi polygon to a plane. The planes are tangent to the sphere at the generating points of the spherical Voronoi diagram. The generating points become the projective centers of the gnomonic projections.

\subsection{The generating points (projective centers)}

The generating points are chosen by the cartographer based on the areas of interest and number of faces desired in the final product. The projection presented here uses seven generating points, one for each continent. A logical choice for the generating points would be the continental centroids. However, there is much debate over the locations of the continental centroids and no reliable published values were found. According to Mitchell B. Adelson of the U.S. Geological Survey, "The concept of where a geographic center is located is rather a simple concept, but it's not that easy to calculate where that centroid is located" (M. B. Adelson, personal communication, November 1, 2006). For now, the generating points have been chosen to be central to each continent, such that the process works within the parameters of the software's gnomonic projection, and with no claim that they are actual continental centroids (Table 1).

Table 1: The generating points (projective centers)

\begin{tabular}{|l|c|c|}
\hline \multicolumn{1}{|c|}{ Continent } & Longitude & Latitude \\
\hline Africa & $17.91^{\circ}$ & $7.46^{\circ}$ \\
\hline Antarctica & $0^{\circ}$ & $-90^{\circ}$ \\
\hline Asia & $94.89^{\circ}$ & $48.61^{\circ}$ \\
\hline Australia & $133.61^{\circ}$ & $-24.49^{\circ}$ \\
\hline Europe & $23.24^{\circ}$ & $56.36^{\circ}$ \\
\hline N. America & $-101.66^{\circ}$ & $46.19^{\circ}$ \\
\hline S. America & $-60.51^{\circ}$ & $-12.39^{\circ}$ \\
\hline
\end{tabular}

\subsection{The polyhedron edges}

For two gnomonic projections to match along a shared edge, the shared edge must be the perpendicular bisector of the line between their projective centers. On the sphere, this edge is a great circle arc. On the plane, this edge is a straight line. The process of determining the locations of these edges for joining multiple gnomonic projections is the equivalent of creating a spherical Voronoi diagram (Voronoi, 1908, as cited in Augenbaum \& Peskin, 1985). The great circle arcs of the spherical Voronoi polygons become the edges of the gnomonically projected polyhedron. Figure 29 shows a set of points and the planar Voronoi diagram they generate. 


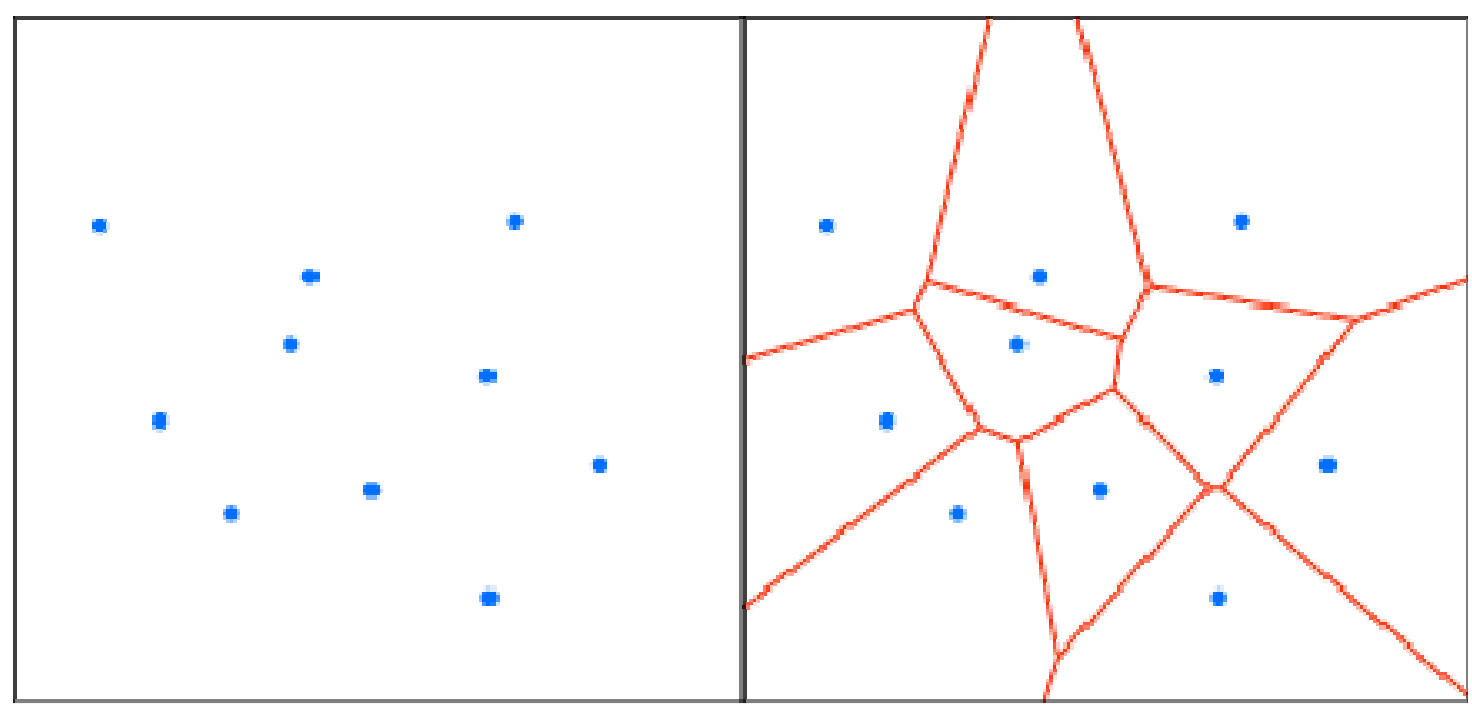

Figure 29. Set of generating points in a plane (left) and the planar Voronoi diagram (right).

Figure 30 shows a set of points on a sphere and the spherical Voronoi diagram they generate.
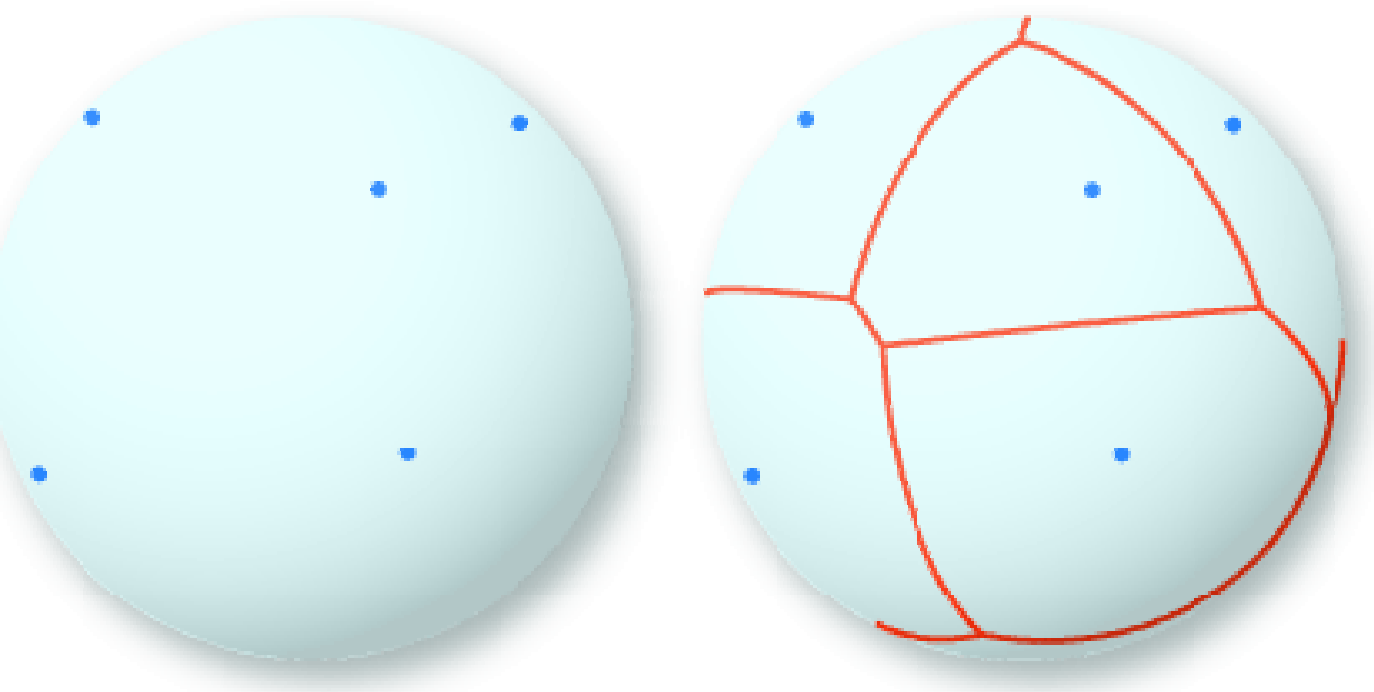

Figure 30. Set of generating points on a sphere (left) and the spherical Voronoi diagram (right).

To define these edges we calculate the vertices, or corner points, that form the spherical Voronoi diagram.

Once all the generating points are chosen, the corner points of the spherical Voronoi polygons can be calculated. A corner point is equidistant from three generating points (Augenbaum \& Peskin, 1985). The generating points need to be expressed in terms of Cartesian coordinates $(x, y, z)$. We convert from geographic coordinates, longitude $(\lambda)$ and latitude $(\phi)$, to Cartesian coordinates on a unit sphere by 


$$
\begin{aligned}
& x=\cos \lambda \cos \phi \\
& y=\sin \lambda \cos \phi \\
& z=\sin \phi
\end{aligned}
$$

(Neutsch, 1996) ${ }^{3}$. If we let $\mathbf{t}, \mathbf{u}$, and $\mathbf{v}$ be the vectors of the three generating points, then the corner point, $\mathbf{c}$, between them is calculated by

$$
\begin{aligned}
\mathbf{c} & =\frac{(\mathbf{u}-\mathbf{t}) \times(\mathbf{v}-\mathbf{t})}{|(\mathbf{u}-\mathbf{t}) \times(\mathbf{v}-\mathbf{t})|} \\
& =\frac{\mathbf{t} \times \mathbf{u}+\mathbf{u} \times \mathbf{v}+\mathbf{v} \times \mathbf{t}}{|\mathbf{t} \times \mathbf{u}+\mathbf{u} \times \mathbf{v}+\mathbf{v} \times \mathbf{t}|}
\end{aligned}
$$

(Augenbaum \& Peskin, 1985). This is the sum of the cross products divided by its norm, which ensures the resulting vector is a unit vector and, hence, describes a point on the surface of the unit sphere. Depending on the ordering of $\mathbf{t}, \mathbf{u}$, and $\mathbf{v}$ in Equations 2 through 5, this equation results in antipodal vertices. The ordering we will use is counterclockwise when viewed from above.

Figure 31 illustrates what is known as the "right-hand rule" (McCallum et al., 1994). If we let the curled fingers on a right hand represent the ordering of the points in the above equations, then the thumb shows on which side of the sphere the resulting point lies.
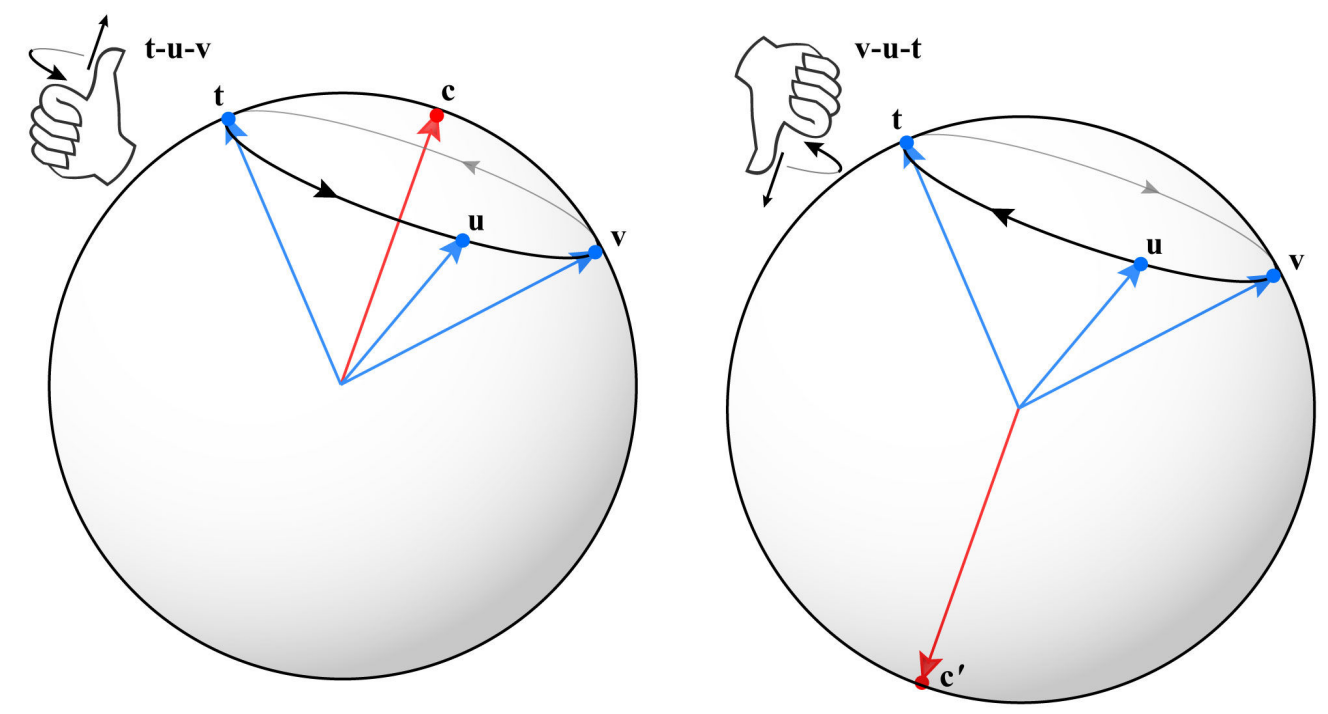

Figure 31. The right-hand rule, showing the effects of order on the vectors.

Using the vector components, Equation 2 becomes

\footnotetext{
${ }^{3}$ Careful attention to the angular units must be paid throughout this process. Trigonometric functions in many software packages expect angles to be expressed in radians, whereas mapping software may expect decimal degrees.
} 


$$
\begin{aligned}
& \left\lfloor\mathbf{x}\left(t_{y} u_{z}-t_{z} u_{y}\right)+\mathbf{y}\left(t_{z} u_{x}-t_{x} u_{z}\right)+\mathbf{z}\left(t_{x} u_{y}-t_{y} u_{x}\right)\right\rfloor \\
& +\left[\mathbf{x}\left(u_{y} v_{z}-u_{z} v_{y}\right)+\mathbf{y}\left(u_{z} v_{x}-u_{x} v_{z}\right)+\mathbf{z}\left(u_{x} v_{y}-u_{y} v_{x}\right)\right] \\
& \mathbf{c}=\frac{+\left[\mathbf{x}\left(v_{y} t_{z}-v_{z} t_{y}\right)+\mathbf{y}\left(v_{z} t_{x}-v_{x} t_{z}\right)+\mathbf{z}\left(v_{x} t_{y}-v_{y} t_{x}\right)\right]}{\left.\| \mathbf{x}\left(t_{y} u_{z}-t_{z} u_{y}\right)+\mathbf{y}\left(t_{z} u_{x}-t_{x} u_{z}\right)+\mathbf{z}\left(t_{x} u_{y}-t_{y} u_{x}\right)\right]} \\
& +\left[\mathbf{x}\left(u_{y} v_{z}-u_{z} v_{y}\right)+\mathbf{y}\left(u_{z} v_{x}-u_{x} v_{z}\right)+\mathbf{z}\left(u_{x} v_{y}-u_{y} v_{x}\right)\right] \\
& +\left[\mathbf{x}\left(v_{y} t_{z}-v_{z} t_{y}\right)+\mathbf{y}\left(v_{z} t_{x}-v_{x} t_{z}\right)+\mathbf{z}\left(v_{x} t_{y}-v_{y} t_{x}\right)\right]
\end{aligned}
$$

Rearranging the elements we have,

$$
\begin{gathered}
\mathbf{x}\left[\left(t_{y} u_{z}-t_{z} u_{y}\right)+\left(u_{y} v_{z}-u_{z} v_{y}\right)+\left(v_{y} t_{z}-v_{z} t_{y}\right)\right] \\
+\mathbf{y}\left[\left(t_{z} u_{x}-t_{x} u_{z}\right)+\left(u_{z} v_{x}-u_{x} v_{z}\right)+\left(v_{z} t_{x}-v_{x} t_{z}\right)\right] \\
\mathbf{c}=\frac{+\mathbf{z}\left[\left(t_{x} u_{y}-t_{y} u_{x}\right)+\left(u_{x} v_{y}-u_{y} v_{x}\right)+\left(v_{x} t_{y}-v_{y} t_{x}\right)\right]}{\mid \mathbf{x}\left[\left(t_{y} u_{z}-t_{z} u_{y}\right)+\left(u_{y} v_{z}-u_{z} v_{y}\right)+\left(v_{y} t_{z}-v_{z} t_{y}\right)\right]} \\
+\mathbf{y}\left[\left(t_{z} u_{x}-t_{x} u_{z}\right)+\left(u_{z} v_{x}-u_{x} v_{z}\right)+\left(v_{z} t_{x}-v_{x} t_{z}\right)\right] \\
+\mathbf{z}\left[\left(t_{x} u_{y}-t_{y} u_{x}\right)+\left(u_{x} v_{y}-u_{y} v_{x}\right)+\left(v_{x} t_{y}-v_{y} t_{x}\right)\right]
\end{gathered} .
$$

Evaluating the norm (see Appendix A) we have,

$$
\begin{aligned}
& \mathbf{x}\left[\left(t_{y} u_{z}-t_{z} u_{y}\right)+\left(u_{y} v_{z}-u_{z} v_{y}\right)+\left(v_{y} t_{z}-v_{z} t_{y}\right)\right] \\
& +\mathbf{y}\left[\left(t_{z} u_{x}-t_{x} u_{z}\right)+\left(u_{z} v_{x}-u_{x} v_{z}\right)+\left(v_{z} t_{x}-v_{x} t_{z}\right)\right]
\end{aligned}
$$

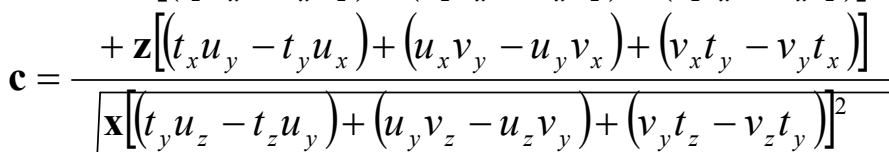

$$
\begin{aligned}
& +\mathbf{y}\left[\left(t_{z} u_{x}-t_{x} u_{z}\right)+\left(u_{z} v_{x}-u_{x} v_{z}\right)+\left(v_{z} t_{x}-v_{x} t_{z}\right)\right]^{2} \\
& 1+\mathbf{z}\left[\left(t_{x} u_{y}-t_{y} u_{x}\right)+\left(u_{x} v_{y}-u_{y} v_{x}\right)+\left(v_{x} t_{y}-v_{y} t_{x}\right)\right]^{2} \text {. }
\end{aligned}
$$

Once the corner point is calculated, it can be converted back to geographic coordinates. To convert from Cartesian coordinates to geographic coordinates we use, 


$$
\begin{aligned}
& \lambda=\left\{\begin{array}{l}
-\pi+\tan ^{-1}\left(\frac{c_{y}}{c_{x}}\right) ; \text { if } c_{x}<0 \text { and } c_{y}<0 \\
\pi+\tan ^{-1}\left(\frac{c_{y}}{c_{x}}\right) ; \text { if } c_{x}<0 \text { and } c_{y}>0 \\
\tan ^{-1}\left(\frac{c_{y}}{c_{x}}\right) ; \text { if } c_{x}>0 \\
\frac{\pi}{2} ; \text { if } c_{x}=0 \text { and } c_{y}=1 \\
-\frac{\pi}{2} ; \text { if } c_{x}=0 \text { and } c_{y}=-1
\end{array}\right. \\
& \phi=\sin ^{-1}\left(c_{z}\right)
\end{aligned}
$$

and then convert from radians to decimal degrees. This process gives a shared vertex of three adjacent Voronoi polygons and must be repeated for all adjacent polygons.

Once all the vertices have been calculated, creating the final projection is a matter of projecting each face using the generating points as the projective centers of a gnomonic projection and clipping along the Voronoi polygon boundaries. The polygon boundaries are great circle arcs on the spherical Voronoi diagram and appear as straight lines between the vertices on a gnomonic projection (Figure 32).
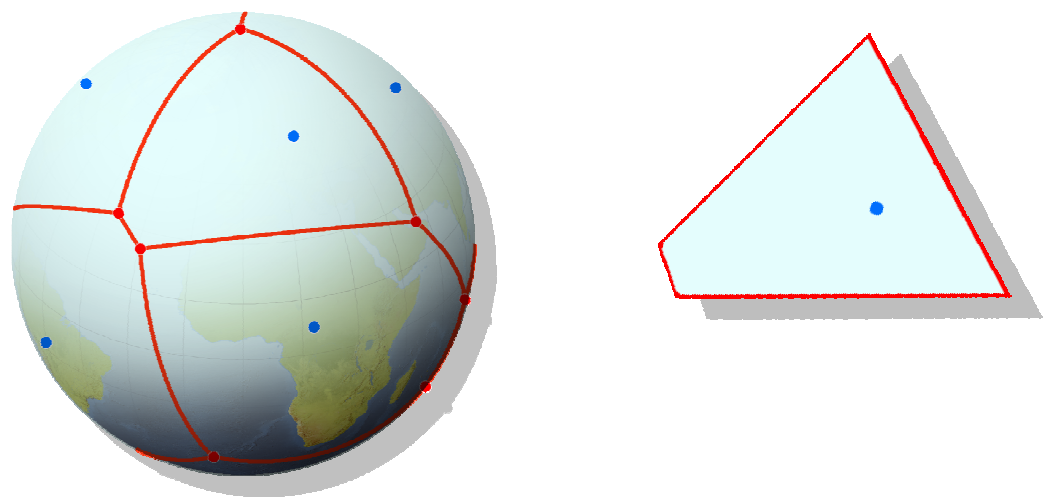

Figure 32. Spherical Voronoi diagram and one of its "flattened" polygons.

Figure 33 shows the assembled irregular heptahedron projection with Voronoi polygon boundaries and the generating points (projective centers).

\footnotetext{
${ }^{4}$ Many programming languages include the function ATAN2 which incorporates the cases in Equation 6.
} 


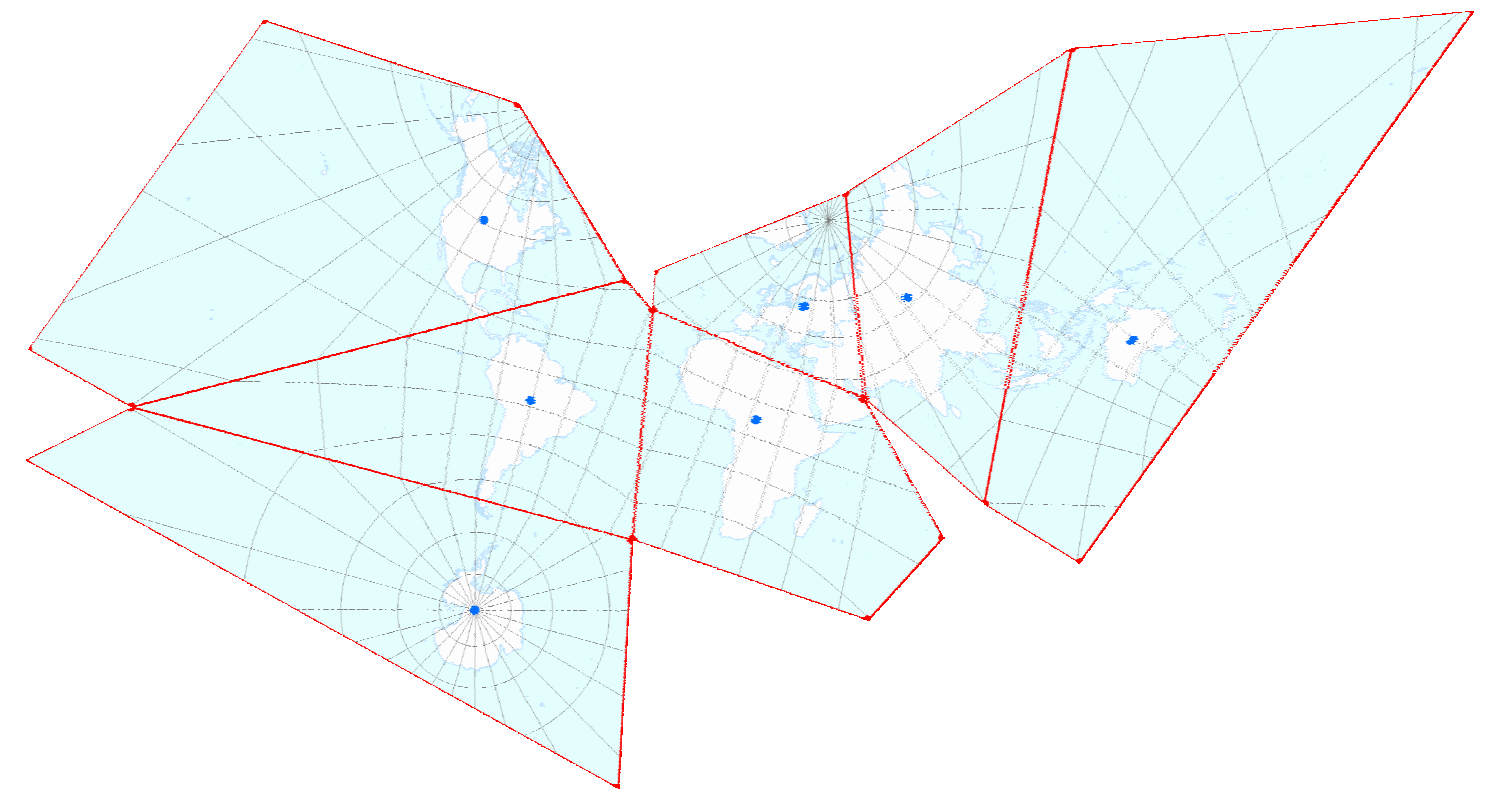

Figure 33: Irregular heptahedron projection with Voronoi polygon boundaries

Figure 34 shows the assembled irregular heptahedron projection without the Voronoi polygon boundaries. The equator is shown in dark gray.

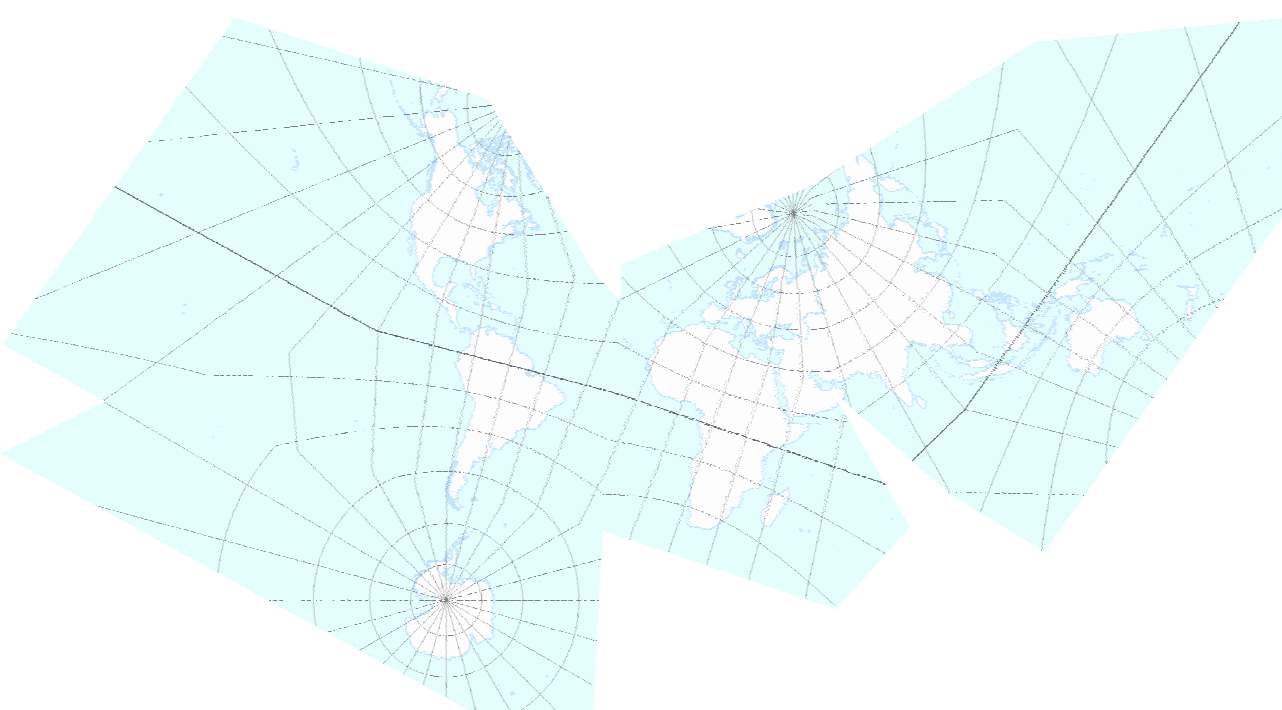

Figure 34. Irregular heptahedron projection. 


\section{Discussion}

Compared to a regular heptahedron projection (Figure 23), we see a great improvement by using an irregular heptahedron. Although the heptahedron-based map in Figure 23 could have been improved by rearranging the net to eliminate some of the breaks in the land masses, the shape distortions would still remain excessive on some of the land masses. This excessive shape distortion results from using inappropriate projective centers and from having to rescale faces to properly match at the edges of the adjacent faces.

Polyhedra with regular faces can introduce distortion by using projective centers that may not be appropriate for the areas of interest being mapped. Their geometry may not provide an appropriate configuration of projective centers to adequately map areas of interest. The method presented here allows the projective centers to be placed anywhere and designs a polyhedron based on a specific arrangement of the areas of interest.

As seen in the example of the regular heptahedron, rescaling faces also introduces distortion. Using a polyhedron that does not have an insphere, meaning its faces are not all tangent to the same sphere, introduces distortion by requiring scale adjustments of the non-tangent faces. This requires extra calculations to determine the scale of the nontangent faces when using the gnomonic projection. The method presented here produces a polyhedron with an insphere, which we take to represent the Earth. Since all faces are tangent to the same sphere, no rescaling is necessary when each face is projected gnomonically. 


\section{Conclusions}

The method presented here for creating a world map projection shows that irregular polyhedra can provide more control over distortion than projections using polyhedra with regular faces. For an irregular polyhedral map, the projective centers can be specified according to the areas of interest, rather than relying on a prearranged geometry as is the case with polyhedra with regular faces. Irregular polyhedra also allow for more control of interruptions within the map, making the map easier to read. The method of creating a Voronoi-partitioned sphere results in a polyhedron with faces that are tangent to a sphere of the same size, ensuring that no scale adjustments are necessary for the assembly of individual projected faces. This method of map projection shows significant potential for creating low distortion world maps. 


\section{Future directions}

Creating a seven-faced irregular polyhedral projection demonstrated potential for this method of creating map projections. Polyhedra with more faces experience a greater reduction in distortion. For example, more than one generating point could be assigned for South America. How many points could one assign for South America? Many. The distortion in South America and other continents could be reduced by adding more generating points and by finding an optimal arrangement of these points. Different versions could be constructed to minimize area or shape distortion. 



\section{REFERENCES}

American Cartographic Association. (1986). Which map is best? Projections for world maps. Special publication no.1. Falls Church, VA: American Congress on Surveying and Mapping.

American Cartographic Association. (1988). Choosing a world map: attributes, distortions, classes, aspects. Special publication no.2. Falls Church, VA: American Congress on Surveying and Mapping.

Augenbaum, J. M., \& Peskin, C. S. (1985). On the construction of the Voronoi mesh on a sphere. Journal of Computational Physics, 59, 177-192.

Buckminster Fuller Institute. (2002). The Fuller Projection: Dymaxion ${ }^{\mathrm{TM}}$ Air-Ocean World. Retrieved February 19, 2006, from http://bfi.org

Cahill, B. J. S. (1909). An account of a new land map of the world. The Scottish Geographical Magazine, 25(9), 449-469.

Cahill, B. J. S. (1913). U.S. Patent No. 1,054,276. Washington, DC: U.S. Patent and Trademark Office. Retrieved December 8, 2006, from http://www.uspto.gov

Cromwell, P. R. (1997). What is regularity? In Polyhedra (p. 77). New York: Cambridge University Press.

Fisher, I., \& Miller, O. M. (1944). World maps and globes. New York: Essential books.

Fuller, R. B. (1946). U.S. Patent No. 2,393,676. Washington, DC: U.S. Patent and Trademark Office. Retrieved December 8, 2006, from http://www.uspto.gov

Goode, J. P. (1925). The homolosine projection: a new device for portraying the Earth's surface entire. Annals of the American Association of Geographers, 15(3), 119125.

Gray, R. W. (1995). Exact transformation equations for Fuller's world map. Cartographica, 32(3), 17-25.

Hafner, I., \& Zitko, T. (n.d.). World's maps on polyhedra nets. Retrieved November 17, 2006, from http://torina.fe.uni-lj.si/ izidor/CuriousMaps/Curious.html

Lay, D. C. (1994). Inner product, length, and orthogonality. In Linear algebra and its applications (p. 334). Reading, MA: Addison-Wesley.

Lethcoe, K. J., \& Klaver, R.W. (1998). Simulating the Interrupted Goode Homolosine Projection with ArcInfo. Paper presented at the 1998 ESRI Users Conference. Retrieved February 18, 2006, from http://gis.esri.com

McCallum, W. G., Hughes-Hallett, D., Gleason, A. M., Gordon, S. P., Thrash, J. B., Osgood, B. G., et al. (1994). The curl of a vector field. In Multivariable calculus (draft version) (p. 417). New York: John Wiley \& Sons.

Neutsch, W. (1996). Coordinates in $\mathfrak{R}^{3}$. In Coordinates (p. 1182). New York: Walter de Gruyter \& Co. 
Snyder, J. P. (1987). Map projections - a working manual. U.S. Geological Survey professional paper 1395. Washington, DC: United States Government Printing Office.

Snyder, J. P. (1992). An equal-area map projection for polyhedral globes. Cartographica, 29(1), 10-21.

Snyder, J. P. (1993). Flattening the earth: Two thousand years of map projections. Chicago: University of Chicago Press.

Weisstein, E. W. (2002). Convex Polyhedron. Mathworld. Retrieved November 17, 2006, from http://mathworld.wolfram.com/ConvexPolyhedron.html

Weisstein, E. W. (2004). Archimedean Solid. Mathworld. Retrieved Decemeber 3, 2006, from http://mathworld.wolfram.com/ArchimedeanSolid.html

Weisstein, E. W. (2004). Insphere. Mathworld. Retrieved January 6, 2007, from http://mathworld.wolfram.com/Insphere.html

Weisstein, E. W. (2005). Spherical Coordinates. Mathworld. Retrieved November 27, from http://mathworld.wolfram.com/SphericalCoordinates.html

Weisstein, E. W. (2006). Johnson solids. Mathworld. Retrieved November 17, 2006, from http://mathworld.wolfram.com/JohnsonSolid.html 


\section{Appendix A - Vector math}

A vector is essentially a list of numbers (Lay, 1994). A pair of geographic coordinates forms a vector that describes a point on the globe. In Cartesian coordinates, a list of three numbers forms a vector that describes a point on the globe.

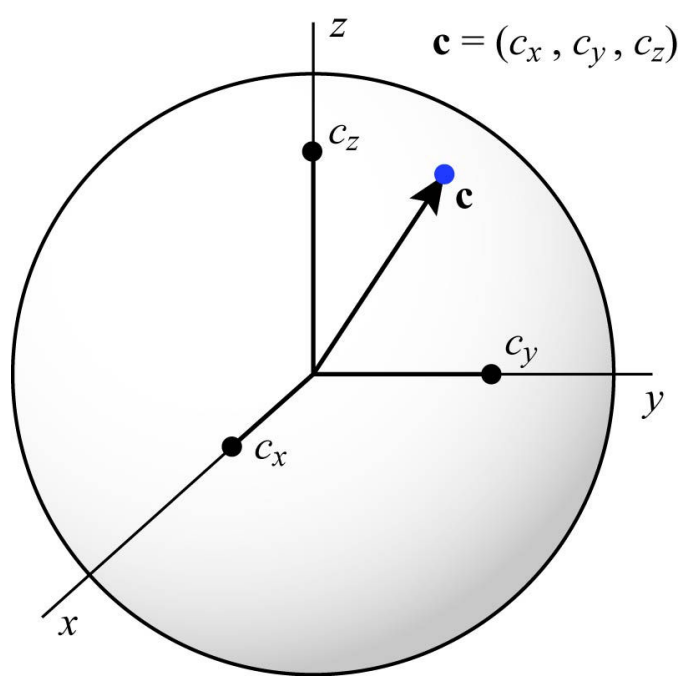

For vectors $\mathbf{u}=\left(u_{x}, u_{y}, u_{z}\right)$ and $\mathbf{v}=\left(v_{x}, v_{y}, v_{z}\right)$ that represent points on a unit sphere, the cross product is defined as

$$
\mathbf{u} \times \mathbf{v}=\left|\begin{array}{ccc}
\mathbf{x} & \mathbf{y} & \mathbf{z} \\
u_{x} & u_{y} & u_{z} \\
v_{x} & v_{y} & v_{z}
\end{array}\right|
$$

(Weisstein, 2005). To calculate the cross product we use,

$$
\begin{aligned}
\mathbf{u} \times \mathbf{v} & =\mathbf{x}\left(u_{y} v_{z}-u_{z} v_{y}\right)-\mathbf{y}\left(u_{x} v_{z}-u_{z} v_{x}\right)+\mathbf{z}\left(u_{x} v_{y}-u_{y} v_{x}\right) \\
& =\mathbf{x}\left(u_{y} v_{z}-u_{z} v_{y}\right)+\mathbf{y}\left(u_{z} v_{x}-u_{x} v_{z}\right)+\mathbf{z}\left(u_{x} v_{y}-u_{y} v_{x}\right)
\end{aligned}
$$

(Weisstein, 2005).

The norm, or length, of a vector is given by $|\mathbf{m}|=\sqrt{m_{1}^{2}+m_{2}^{2}+\cdots+m_{n}^{2}}$ (Lay, 1994). In our case we have $|\mathbf{m}|=\sqrt{m_{x}^{2}+m_{y}^{2}+m_{z}^{2}}$. 


\section{Appendix B - The process in ArcGIS ${ }^{\circledR}$}

A personal geodatabase was created. Then, the continents shapefile and world 15 grid shapefiles were imported into the newly created personal geodatabase. In ArcMap, the Excel .csv file containing the $\mathrm{x}$, $\mathrm{y}$ data for the ten Voronoi vertices was added. The data were added to the map with the "Display XY Events..." command. The vertices were then exported to a featureclass in the personal geodatabase. The coordinate system for the data frame was then set to a polar gnomonic projection, modified to be centered at one of the Voronoi generating points. A polygon feature class was created with the same spatial reference as the data frame. This polygon feature class was created for the boundary polygon. The boundary polygon for this face was digitized by snapping to the corresponding points in the Voronoi vertices feature class. Then both the continents and world 15 feature classes were clipped using this boundary polygon to new feature classes with the same spatial reference as the boundary polygon feature class. Clipping these feature classes to new feature classes with the appropriate spatial reference eliminates printing problems, such as some data not printing. Seven data frames were created using this process.

Once all seven data frames were created, they were arranged in layout mode (Figure 34).

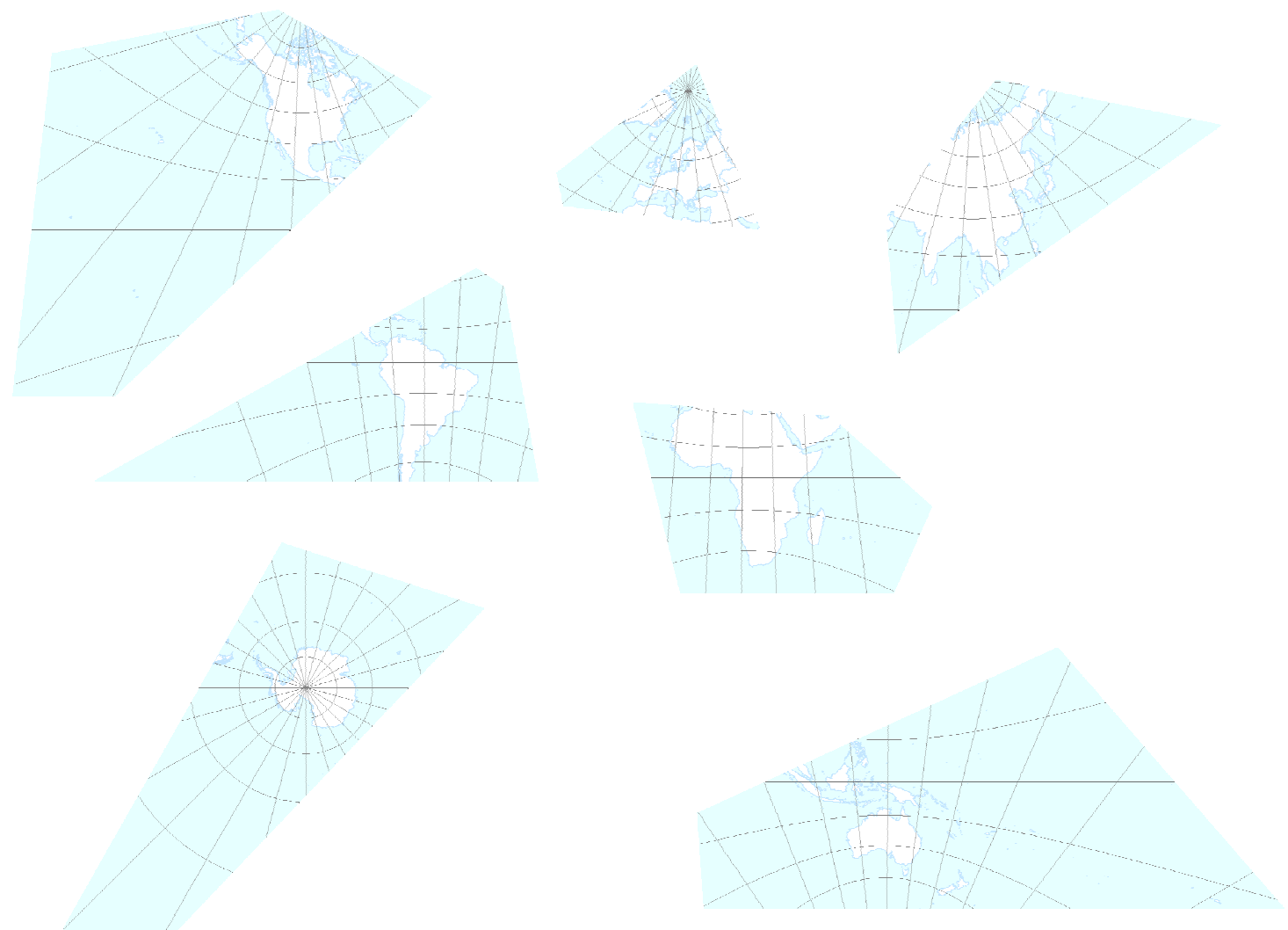

Figure 35. Layout view of the seven data frames in ArcMap ${ }^{\mathrm{TM}}$

The "Data Frame Tools" toolbar was necessary to rotate the data frames. All data frames were set to the same scale. The method presented here eliminates the need for some 
faces to be rescaled in order to match adjacent faces which can be the case when creating a polyhedron map projection. Each face was rotated and positioned to join its neighboring face. 